\title{
A Hierarchical Uniformly High Order DG-IMEX Scheme for the 1D BGK Equation ${ }^{1}$
}

\author{
Tao Xiong ${ }^{2}$ and Jing-Mei Qiu ${ }^{3}$
}

\begin{abstract}
A class of high order nodal discontinuous Galerkin implicit-explicit (DG-IMEX) schemes with asymptotic preserving (AP) property has been developed for the one-dimensional (1D) BGK equation in Xiong et. al. (2015) [40], based on a micro-macro reformulation. The schemes are globally stiffly accurate and asymptotically consistent, and as the Knudsen number becomes small or goes to zero, they recover first the compressible Navier-Stokes (CNS) and then the Euler limit. Motivated by the recent work of Filbet and Rey (2015) [27] and references therein, in this paper, we propose a hierarchical high order AP method, namely kinetic, CNS and Euler solvers are automatically applied in regions where their corresponding models are appropriate. The numerical solvers for different regimes are coupled naturally by interface conditions. To the best of our knowledge, the resulting scheme is the very first hierarchical one being proposed in the literature, that enjoys AP property as well as uniform high order accuracy. Numerical experiments demonstrate the efficiency and effectiveness of the proposed approach. As time evolves, three different regimes are dynamically identified and naturally coupled, leading to significant CPU time savings (more than $80 \%$ for some of our test problems).
\end{abstract}

Keywords: A hierarchical scheme, Compressible Euler equations, Compressible Navier-Stokes equations, Asymptotic Preserving, Nodal Discontinuous Galerkin, High order, IMEX, BGK equation

\footnotetext{
${ }^{1}$ Research supported by NSF DMS-1217008, DMS-1522777 and Air Force Office of Scientific Computing FA9550-16-1-0179, the Fundamental Research Funds for the Central Universities No. 20720160009, NSFC grant 11601455, NSAF grant U1630247 and NSF grant of Fujian Province 2016J05022, and the Marie SkłodowskaCurie Individual Fellowships H2020-MSCA-IF-2014 of the European Commision, under the project HNSKMAP 654175 .

${ }^{2}$ School of Mathematical Sciences, Fujian Provincial Key Laboratory of Mathematical Modeling and HighPerformance Scientific Computing, Xiamen University, Xiamen, Fujian, P.R. China, 361005. Currently visiting at the Université de Toulouse III, UMR 5219, Institut de Mathématiques de Toulouse, 118 route de Narbonne, F-31062 Toulouse cedex, France. Email: txiong@xmu.edu.cn

${ }^{3}$ Department of Mathematics, University of Houston, Houston, 77004. E-mail: jingqiu@math.uh.edu.
}

(C) 2017. This manuscript version is made available under the Elsevier user license http://www.elsevier.com/open-access/userlicense/1.0/ 


\section{Introduction}

In physics, rarefied gases can be described by kinetic models using the Boltzmann equation. In such a description, Knudsen number $\varepsilon$ is an important dimensionless parameter, defined as the ratio of the molecular mean free path length to a representative physical length scale, characterizing the frequency of molecular collisions or how rarefied the gas is. In the zero limit of Knudsen number, the compressible Euler system describing the conservation of mass, momentum and energy is a sufficient macroscopic model, while when the Knudsen is sufficiently small but not zero, the compressible Navier-Stokes (CNS) equations with viscosity and heat conductivity terms are needed. BGK equation is a simplified model for the Boltzmann equation introduced by Bhatnagar, Gross and Krook [9].

Many numerical schemes have been proposed for solving the BGK and Boltzmann equations with a wide range of Knudsen number. A micro-macro decomposition framework was proposed by Bennoune, Lemou, Mieussen [8], which can correctly capture the macroscopic NavierStokes limit when the Knudsen number is sufficiently small. Various versions of implicit-explicit schemes were proposed for the BGK equations in [33, 34] and for the ellipsoidal statistics BGK (ES-BGK) equation in [26]. A BGK-penalization strategy was proposed by Filbet and Jin [25] for the Boltzmann equation. These methods all belong to the class of asymptotic preserving (AP) schemes, which are designed to mimic the asymptotic limit from a kinetic to a hydrodynamic model on the PDE level as $\varepsilon$ goes to 0 [29]. In particular, there is the asymptotical consistency, meaning that the numerical scheme in the asymptotic limit becomes a consistent discretization of the limiting equations. Associated with the AP concept, there is also the asymptotically accurate property, indicating that the numerical scheme in the asymptotic limit becomes a high order accurate discretization of the limiting system. Designing high order schemes with the asymptotic accurate property is usually a very challenging task, considering different nature of the equation systems for multi-scale problems.

A family of high order AP schemes for the BGK equation has been developed in [40], based on the micro-macro decomposition framework. The proposed methods work for both constant Knudsen number $\varepsilon$ and spatially variant $\varepsilon=\varepsilon(x)$ ranging from zero to order one. The high order spatial accuracy is achieved by nodal discontinuous Galerkin (NDG) finite element approaches [28], and the high order temporal accuracy is achieved by globally stiffly accurate implicitexplicit (IMEX) Runge-Kutta (RK) methods [10, 11]. A formal asymptotic analysis shows that the scheme becomes a DG method [36] or an local DG (LDG) [5, 18, 7, 31, 6] method with explicit RK time discretizations for the compressible Euler or CNS systems, in the zero limit of Knudsen number or when the Knudsen number becomes sufficiently small (but not zero), respectively. In other words, the NDG IMEX schemes are asymptotically accurate for both the Euler and CNS limits. In this paper, we propose to build a hierarchical high order scheme based on the above mentioned asymptotically accurate NDG IMEX schemes.

Kinetic and fluid models represent complementary approaches for the description of a given physical problem: kinetic models, on the one hand, are more accurate but also computationally very expensive, due to the extra three dimensions required by the phase space formulation; fluid models such as Euler and CNS systems, on the other hand, have a lower computational cost but typically break down near shocks or kinetic boundary layers. In a multi-scale scenario, it is of interest to use the kinetic solver only locally in regions where it is necessary, while taking advantage of low computational cost of fluid solvers elsewhere. In fact, many hybrid kinetic/fluid schemes with static or dynamic domain decomposition criteria have been under 
great development. For example, there are criteria proposed by Boyd, Chen and Candler based on a local Knudsen number represented by gradients of macroscopic variables [12]. There are criteria derived from Grad's 13-moments expansion methods proposed by Tiwari [37], as well as the moment realizability criteria introduced by Levermore, Morokoff and Nadiga [30]. With proper decomposition of a computational domain into kinetic and hydrodynamic regimes, hybrid schemes typically couple kinetic solvers with fluid ones with proper interface coupling conditions to pass information between those regimes. Examples of such hybrid schemes include the coupling of a discrete velocity model [21, 1] or a Monte Carlo method [37, 24, 19] for the kinetic model, together with a finite volume method for the Euler system [24] or a smoothedparticle hydrodynamics (SPH) method for the CNS system [39, 38]. In a sequence of work in $[22,20,21]$, the authors proposed and applied a smoothly varying transition function $h$ that automatically and dynamically creates buffer zones to ensure smooth transition of solvers for kinetic and fluid regimes. More recently, several hybrid schemes have been proposed based on an equilibrium and non-equilibrium decomposition of the distribution function [21, 24] based on the work in [23], leading to an automatic preservation of the uniform flow [22].

In this paper, we propose to couple several state-of-the-art ingredients, such as the high order AP scheme formulation, regime identification criteria and interface coupling conditions. In particular, we propose a hierarchical scheme based on the NDG-IMEX schemes developed in [40]. Thanks to the AP framework and asymptotically accurate properties of the NDG-IMEX schemes, the domain decomposition can be naturally applied. It results in a kinetic solver for the full kinetic equation, and automatically becomes a DG scheme for the compressible Euler equations or an LDG scheme for the CNS system. Criteria to adaptively identify three different regimes (kinetic, CNS and Euler) are proposed based on the moment realizability ones introduced in [30] and recently being used in [27]. Seamless interface coupling conditions are proposed and numerically verified to be robust and effective in all of our test examples. Significant savings on the CPU time are observed, as compared to the full NDG-IMEX scheme for the kinetic BGK equation. Note that many existing hybrid methods adaptively select solvers of different nature (e.g. a mesh-based finite volume method and a particle-based method) and couple them together. Comparing with these methods, our proposed solver combines the AP property with the domain decomposition idea, which offers many advantages such as ease and natural setting of interface coupling conditions, automatic preservation of uniform flow.

We highlight a few good properties of our proposed hierarchical scheme, compared with existing state-of-the-art hybrid domain decomposition methods in the literature. (1) Our scheme applies a finer classification of three different regimes with three high order solvers in space and in time, i.e. NDG-IMEX, LDG-RK and DG-RK for kinetic, CNS and Euler models, respectively. The three high order solvers are consistent with each other in the sense that the NDG-IMEX solver for the kinetic equation is reduced to the LDG-RK or DG-RK solvers for CNS and Euler automatically as the Knudsen number becomes small or approaches zero (by formal asymptotic analysis). (2) Thanks to the compactness property of the DG scheme, all of our three solvers are very compact. Therefore, no transitional region (e.g. the $h$ function introduced in [22]) is needed. (3) Due to the micro-macro decomposition mechanism we used, our scheme automatically preserves the uniform flow as in [21]. (4) No operator or dimensional splitting is used in our scheme formulation. Therefore, no splitting error is involved. Finally, we remark that our scheme is similar to the one proposed in [21] in the sense that we both use the micro-macro decomposition of the system. Besides our scheme is high order in space and in time via the DG formulation and IMEX schemes for three different regimes (i.e. kinetic, CNS 
and Euler). Due to the compactness of the DG formulation, we do not involve the transition function $h$ (i.e. buffer zones between fluid and kinetic regions) as in [21].

The rest of the paper is organized as follows. In Section 2, the BGK equation in a hyperbolic scaling and its micro-macro decomposition is given. In Section 3, high order AP nodal DG spatial discretization and globally stiffly accurate IMEX temporal discretizations are presented. The regime indicators are introduced. In Section 4, numerical results are performed for one dimensional problems. Conclusions are given in the final section.

\section{BGK Equation and Micro-macro Formulation}

We consider the BGK equation in a hyperbolic scaling [9]:

$$
\partial_{t} f+v \cdot \nabla_{x} f=\frac{1}{\varepsilon}\left(M_{U}-f\right)
$$

where $f=f(x, v, t)$ is the distribution function of particles that depends on time $t>0$, position $x \in \Omega_{x} \subset \mathbb{R}^{d}$ and velocity $v \in \mathbb{R}^{d}$ for $d \geq 1$. The parameter $\varepsilon$ is the Knudsen number proportional to the mean free path, and $M_{U}$ is the local Maxwellian defined by

$$
M_{U}=M_{U}(x, v, t)=\frac{\rho(x, t)}{(2 \pi T(x, t))^{d / 2}} \exp \left(-\frac{|v-u(x, t)|^{2}}{2 T(x, t)}\right) .
$$

$\rho, u, T$ represent the macroscopic density, the mean velocity, and the temperature respectively. $U$ has the components of the density, momentum and energy, which are obtained by taking the first three moments of $f$ :

$$
U:=(\rho, \rho u, E)^{\top}=\int_{\mathbb{R}^{d}}\left(1, v, \frac{1}{2}|v|^{2}\right)^{\top} f(v) d v
$$

where $E=\frac{1}{2} \rho|u|^{2}+\frac{d}{2} \rho T$ and the superscript $T$ denotes the transpose of the corresponding vector. In this paper, we use $m=m(v):=\left(1, v, \frac{1}{2}|v|^{2}\right)^{\top}$ and let $\langle g\rangle:=\int_{\mathbb{R}^{d}} g(v) d v$. It is easy to check that $\left\langle m M_{U}\right\rangle=\left(\rho, \rho u, \frac{1}{2} \rho|u|^{2}+\frac{d}{2} \rho T\right)^{\top}=U$. Hence $\left\langle m\left(M_{U}-f\right)\right\rangle=0$ and

$$
\partial_{t} U+\nabla_{x}\langle v m f\rangle=0
$$

namely the BGK operator $M_{U}-f$ satisfies the conservation of mass, momentum and energy. Moreover, it enjoys the entropy dissipation: $\left\langle\left(M_{U}-f\right) \log f\right\rangle \leq 0$.

In the following, we briefly recall the micro-macro decomposition of (2.1), from which the compressible Euler and Navier-Stokes limits will follow. For details, see [40]. Let us first introduce several notations. Taking $M=M_{U}$ for short, we use $L_{M}^{2}$ to denote the Hilbert space equipped with the weighted inner product

$$
\left(\beta_{1}, \beta_{2}\right)_{M}:=\left\langle\beta_{1} \beta_{2} M^{-1}\right\rangle,
$$

for any function $\beta_{1}(v), \beta_{2}(v) \in L_{M}^{2}$. Then we can write $f(x, \cdot, t) \in L_{M}^{2}$ as

$$
f=\Pi_{M} f+\left(\mathbf{I}-\Pi_{M}\right) f .
$$


where $\Pi_{M} f$ is an orthogonal projection from $L_{M}^{2}$ onto $\mathcal{N}:=\operatorname{span}\left\{M, v M,|v|^{2} M\right\}$, and it is explicitly given by

$$
\Pi_{M} f=\left(\frac{1}{\rho}\langle f\rangle+\frac{\langle(v-u) f\rangle}{\rho T} \cdot(v-u)+\frac{2}{d \rho}\left\langle\left(\frac{|v-u|^{2}}{2 T}-\frac{d}{2}\right) f\right\rangle\left(\frac{|v-u|^{2}}{2 T}-\frac{d}{2}\right)\right) M .
$$

For micro-macro reformulation, we start with decomposing $f$ into a macroscopic part $M$ and a microscopic part $\varepsilon g$,

$$
f:=M+\varepsilon g
$$

with $\langle m g\rangle=0$. Then for constant $\varepsilon$, inserting (2.5) into (2.1) and applying the orthogonal projections $\Pi_{M}$ and $\mathbf{I}-\Pi_{M}$ respectively, we will have the following micro-macro decomposed equations:

$$
\begin{aligned}
& \partial_{t} U+\nabla_{x} \cdot F(U)+\varepsilon \nabla_{x} \cdot\langle v m g\rangle=0 \\
& \varepsilon \partial_{t} g+\varepsilon\left(\mathbf{I}-\Pi_{M}\right)\left(v \cdot \nabla_{x} g\right)=-\left(g+\left(\mathbf{I}-\Pi_{M}\right)\left(v \cdot \nabla_{x} M\right)\right)
\end{aligned}
$$

where the flux $F(U)=(\rho u, \rho u \otimes u+p I,(E+p) u)^{\top}$ and $p=\rho T . I$ is the $d \times d$ identity matrix. In a more general setting where the Knudsen number depends on the position $\varepsilon=\varepsilon(x)$, the micro-macro formulation (2.6) should be written as follows:

$$
\begin{aligned}
& \partial_{t} U+\nabla_{x} \cdot F(U)+\nabla_{x} \cdot(\varepsilon(x)\langle v m g\rangle)=0, \\
& \varepsilon(x) \partial_{t} g+\left(\mathbf{I}-\Pi_{M}\right) \nabla_{x} \cdot(\varepsilon(x) v g)=-\left(g+\left(\mathbf{I}-\Pi_{M}\right)\left(v \cdot \nabla_{x} M\right)\right) .
\end{aligned}
$$

We observe that the first two terms in (2.6a) form the Euler system and eq. (2.6a) formally converges to the Euler system as $\varepsilon \rightarrow 0$. For the third term in eq. (2.6a), the leading order $(\varepsilon$ term) will give rise to the viscous term in compressible Navier-Stokes equations. To see this, we have, from $(2.6 \mathrm{~b})$,

$$
g=-\left(\mathbf{I}-\Pi_{M}\right)\left(v \cdot \nabla_{x} M\right)+\mathcal{O}(\varepsilon)
$$

and the direct computation shows that

$$
\frac{\left(\mathbf{I}-\Pi_{M}\right)\left(v \cdot \nabla_{x} M\right)}{M}=\frac{1}{2} A:\left(\nabla_{x} u+\left(\nabla_{x} u\right)^{\top}-\frac{2}{d}\left(\nabla_{x} \cdot u\right) I\right)+B \cdot \frac{\nabla_{x} T}{\sqrt{T}}
$$

where

$$
A=\frac{(v-u) \otimes(v-u)}{T}-\frac{|v-u|^{2}}{d T} I \text { and } B=\left(\frac{|v-u|^{2}}{2 T}-\frac{d+2}{2}\right) \frac{v-u}{\sqrt{T}} .
$$

Therefore, we deduce that

$$
g=-A:\left(\nabla_{x} u+\left(\nabla_{x} u\right)^{\top}-\frac{2}{d}\left(\nabla_{x} \cdot u\right) I\right) M-B \cdot \frac{\nabla_{x} T}{\sqrt{T}} M+\mathcal{O}(\varepsilon) .
$$

Here $X: Y=\sum_{i, j} X_{i j} Y_{i j}$ is the Frobenius inner product for matrices. As we insert the expression (2.11) into (2.6a), we obtain

$$
\partial_{t}\left(\begin{array}{c}
\rho \\
\rho u \\
E
\end{array}\right)+\nabla_{x} \cdot\left(\begin{array}{c}
\rho u \\
\rho u \otimes u+p I \\
(E+p) u
\end{array}\right)=\varepsilon\left(\begin{array}{c}
0 \\
\nabla_{x} \cdot \sigma \\
\nabla_{x} \cdot(\sigma u+q)
\end{array}\right)+\mathcal{O}\left(\varepsilon^{2}\right)
$$


where

$$
\sigma=\mu D(u), \quad D(u)=\nabla_{x} u+\left(\nabla_{x} u\right)^{\top}-\frac{2}{d}\left(\nabla_{x} \cdot u\right) I \text { and } q=\kappa \nabla_{x} T
$$

and

$$
\mu=\frac{T}{4}\langle A: A M\rangle \text { and } \kappa=T\langle B \cdot B M\rangle
$$

We refer to [4] for more details on the derivation. The above system (2.12) is the compressible Navier-Stokes equations if we disregard high order terms $\mathcal{O}\left(\varepsilon^{2}\right)$. We note that when $d=1$, $\sigma=0$ and $\kappa=\frac{3}{2} \rho T$.

As we point out in [40], although the BGK equation shares the basic properties of hydrodynamics with the Boltzmann equation, the Navier-Stokes equations derived from those equations display different viscosity and heat conductivity coefficients $[14,15,16]$. We would remark that in this case, the ES-BGK [2] operator can be used in the micro-macro decomposition framework.

\section{$3 \quad$ NDG-IMEX Methods}

In this section, we will briefly review the NDG-IMEX scheme developed in [40], where the nodal discontinuous Galerkin (NDG) method in space together with implicit-explicit (IMEX) Runge-Kutta $(\mathrm{RK})$ time discretization is used. We will only focus on the one-dimensional case with $d=1, \Omega_{x}=[a, b]$ and $\Omega_{v}=\left[-V_{c}, V_{c}\right]$ with $V_{c}$ sufficiently large so that the Maxwellian defined in (2.2) can be regarded as zero outside $\Omega_{v}$ numerically. For simplicity, we will just consider Scheme II in [40] for the general $\varepsilon(x)$ in (2.7) here, but Scheme I can be used similarly. Extension to high dimensions will be considered later and contribute to our future work.

\subsection{Semi-discrete NDG methods}

Start with a partition of $\Omega_{x}, a=x_{\frac{1}{2}}<x_{\frac{3}{2}}<\cdots<x_{N_{x}+\frac{1}{2}}=b$. Let $I_{i}=\left[x_{i-\frac{1}{2}}, x_{i+\frac{1}{2}}\right]$ denote an element with its length $h_{i}$, and let $h=\max _{i=0}^{N_{x}} h_{i}$. Given any non-negative integer $K$, we define a finite dimensional discrete space,

$$
Z_{h}^{K}=\left\{z \in L^{2}\left(\Omega_{x}\right):\left.z\right|_{I_{i}} \in P^{K}\left(I_{i}\right), \forall i\right\}
$$

and its vector version is denoted as $\mathbf{Z}_{h}^{K}$. The local space $P^{K}(I)$ consists of polynomials of degree at most $K$ on $I$. Note that functions in $Z_{h}^{K}$ are piecewise defined. To distinguish the left and right limits of a function $z \in Z_{h}^{K}$ at a grid point $x_{i+\frac{1}{2}}$, we let $z_{i+\frac{1}{2}}^{ \pm}=\lim _{\Delta x \rightarrow \pm 0} z\left(x_{i+\frac{1}{2}}+\Delta x\right)$, and we also let $[z]_{i+\frac{1}{2}}=z_{i+\frac{1}{2}}^{+}-z_{i+\frac{1}{2}}^{-}$as the jump.

Consider the relation (2.9) in one dimension, we have

$$
\left(\mathbf{I}-\Pi_{M}\right)\left(v \partial_{x} M\right)=A \frac{\partial_{x} T}{\sqrt{T}} M
$$

With this, the equation $(2.7 \mathrm{~b})$ becomes

$$
\varepsilon(x) \partial_{t} g+\left(\mathbf{I}-\Pi_{M}\right) \partial_{x}(\varepsilon(x) v g)=-\left(g+A \frac{\partial_{x} T}{\sqrt{T}} M\right) .
$$


Keeping $(t, v)$ continuous, a semi-discrete DG method in $x$ for the micro-macro system (2.7) is designed as follows: We seek $U_{h}(\cdot, t) \in \mathbf{Z}_{h}^{K}$ and $g_{h}(\cdot, v, t) \in Z_{h}^{K}$, such that $\forall \phi, \psi \in Z_{h}^{K}$ and $\forall i$,

$$
\begin{aligned}
\int_{I_{i}} \partial_{t} U_{h} \phi d x= & \int_{I_{i}}\left(F\left(U_{h}\right)+\varepsilon(x)\left\langle v m g_{h}\right\rangle\right) \frac{d \phi(x)}{d x} d x-\hat{F}_{i+\frac{1}{2}} \phi_{i+\frac{1}{2}}^{-}+\hat{F}_{i-\frac{1}{2}} \phi_{i-\frac{1}{2}}^{+} \\
& \left.\left.-\varepsilon\left(x_{i+\frac{1}{2}}\right) \widehat{\left\langle v m g_{h}\right.}\right\rangle_{i+\frac{1}{2}} \phi_{i+\frac{1}{2}}^{-}+\varepsilon\left(x_{i-\frac{1}{2}}\right) \widehat{\left\langle v m g_{h}\right.}\right\rangle_{i-\frac{1}{2}} \phi_{i-\frac{1}{2}}^{+} \\
\int_{I_{i}} \varepsilon(x) \partial_{t} g_{h} \psi d x+ & \int_{I_{i}}\left(\mathbf{I}-\Pi_{M_{h}}\right)\left(\mathcal{D}_{h, 1}\left(\varepsilon(x) v g_{h}\right)\right) \psi d x=-\int_{I_{i}} g_{h} \psi d x-\int_{I_{i}} A_{h} \frac{r_{h}}{\sqrt{T_{h}}} M_{h} \psi d x
\end{aligned}
$$

Here $M_{h}=M_{U_{h}}$ according to $(2.2) . \mathcal{D}_{h, 1}\left(\varepsilon(x) v g_{h}\right)(\cdot, v, t) \in Z_{h}^{K}$ and $r_{h} \in Z_{h}^{K}$ are approximations of the spatial derivative of $\varepsilon(x) v g$ and $T$, respectively, based on DG discretizations. Particularly, $\forall \psi \in Z_{h}^{K}$ and $\forall i$

$$
\int_{I_{i}} \mathcal{D}_{h, 1}\left(\varepsilon(x) v g_{h}\right) \psi d x:=-\int_{I_{i}} \varepsilon(x) v g_{h} \frac{d \psi}{d x} d x+\varepsilon\left(x_{i+\frac{1}{2}}\right) \widetilde{\left(v g_{h}\right)_{i+\frac{1}{2}}} \psi_{i+\frac{1}{2}}^{-}-\varepsilon\left(x_{i-\frac{1}{2}}\right) \widetilde{\left(v g_{h}\right)_{i-\frac{1}{2}}} \psi_{i-\frac{1}{2}}^{+},
$$

where $\widetilde{v g}$ is an upwind numerical flux consistent to $v g$,

$$
\widetilde{v g}:= \begin{cases}v g^{-}, & \text {if } v>0 \\ v g^{+}, & \text {if } v<0\end{cases}
$$

and $\forall \varphi \in Z_{h}^{K}$ and $\forall i$

$$
\int_{I_{i}} r_{h} \varphi d x=-\int_{I_{i}} T_{h} \frac{d \varphi}{d x} d x+\widehat{T}_{h, i+\frac{1}{2}} \varphi_{i+\frac{1}{2}}^{-}-\widehat{T}_{h, i-\frac{1}{2}} \varphi_{i-\frac{1}{2}}^{+}
$$

Here $T_{h}$, a macroscopic quantity, and $A_{h}$ can be obtained from $U_{h}$ based on the energy $E$ under (2.3) and (2.10) respectively. The numerical flux $\hat{F}=\hat{F}\left(U_{h}^{-}, U_{h}^{+}\right)$in (3.4a) is taken to be the global Lax-Friedrichs flux [17]. Here the subscripts $i \pm \frac{1}{2}$ are temporarily omitted for simplicity. We take the hatted fluxes $\widehat{\langle v m g\rangle}$ and $\widehat{T}$ as the central fluxes $\widehat{\langle v m g\rangle}=\left\langle v m\left(g^{-}+g^{+}\right)\right\rangle / 2, \widehat{T}=$ $\left(T^{+}+T^{-}\right) / 2$, while the alternating right-left and left-right fluxes introduced in [40] can also be used.

We further use the nodal basis to represent functions in the discrete space $Z_{h}^{K}$, and approximating the integrals in the schemes by numerical quadratures. Note that the discrete space $\left.Z_{h}^{K}\right|_{I_{i}}$ is simply $P^{K}\left(I_{i}\right)$. We particularly choose the local nodal basis (also called Lagrangian basis) $\left\{\phi_{i}^{k}(x)\right\}_{k=0}^{K}$ associated with the $K+1$ Gaussian quadrature points $\left\{x_{i}^{k}\right\}_{k=0}^{K}$ on $I_{i}$, defined as below

$$
\phi_{i}^{k}(x) \in P^{K}\left(I_{i}\right), \quad \text { and } \quad \phi_{i}^{k}\left(x_{i}^{k^{\prime}}\right)=\delta_{k k^{\prime}}, \quad k, k^{\prime}=0,1, \cdots, K .
$$

Here $\delta_{k k^{\prime}}$ is the Kronecker delta function. We further let $\left\{\omega_{k}\right\}_{k=0}^{K}$ denote the corresponding quadrature weights on the reference element $\left(-\frac{1}{2}, \frac{1}{2}\right)$.

With the nodal basis functions, (3.4)-(3.5) and (3.7) can be equivalently stated with the test functions $\phi, \psi$ both being taken as $\phi_{i}^{k}, k=0,1, \cdots, K$. We also replace all the integral terms in (3.4)-(3.5) and (3.7) by their numerical integrations based on $(K+1)$-point Gaussian 
quadrature. The scheme now becomes: find $U_{h}(\cdot, t) \in \mathbf{Z}_{h}^{K}$ and $g_{h}(\cdot, v, t) \in Z_{h}^{K}$, with $\left.U_{h}(x, t)\right|_{I_{i}}=$ $\sum_{k=0}^{K} U_{i}^{k}(t) \phi_{i}^{k}(x),\left.g_{h}(x, v, t)\right|_{I_{i}}=\sum_{k=0}^{K} g_{i}^{k}(v, t) \phi_{i}^{k}(x)$, such that $\forall i, k$,

$$
\begin{aligned}
& \omega_{k} h_{i} \frac{d U_{i}^{k}}{d t}=\left.\sum_{k^{\prime}=0}^{K} \omega_{k^{\prime}} h_{i} F\left(U_{i}^{k^{\prime}}\right) \frac{d \phi_{i}^{k}(x)}{d x}\right|_{x=x_{i}^{k^{\prime}}}-\hat{F}_{i+\frac{1}{2}} \phi_{i}^{k}\left(x_{i+\frac{1}{2}}^{-}\right)+\hat{F}_{i-\frac{1}{2}} \phi_{i}^{k}\left(x_{i-\frac{1}{2}}^{+}\right) \\
& \left.+\left.\sum_{k^{\prime}=0}^{K} \omega_{k^{\prime}} h_{i} \varepsilon\left(x_{i}^{k^{\prime}}\right)\left\langle v m g_{i}^{k^{\prime}}\right\rangle \frac{d \phi_{i}^{k}(x)}{d x}\right|_{x=x_{i}^{k^{\prime}}}-\varepsilon\left(x_{i+\frac{1}{2}}\right) \widehat{\left\langle v m g_{h}\right.}\right\rangle_{i+\frac{1}{2}} \phi_{i}^{k}\left(x_{i+\frac{1}{2}}^{-}\right) \\
& \left.+\varepsilon\left(x_{i-\frac{1}{2}}\right) \widehat{\left\langle v m g_{h}\right.}\right\rangle_{i-\frac{1}{2}} \phi_{i}^{k}\left(x_{i-\frac{1}{2}}^{+}\right), \\
& \varepsilon\left(x_{i}^{k}\right) \omega_{k} h_{i} \partial_{t} g_{i}^{k}=\left(\mathbf{I}-\Pi_{i}^{k}\right)\left(\left.v \sum_{k^{\prime}=0}^{K} \omega_{k^{\prime}} h_{i} \varepsilon\left(x_{i}^{k^{\prime}}\right) g_{i}^{k^{\prime}} \frac{d \phi_{i}^{k}(x)}{d x}\right|_{x=x_{i}^{k^{\prime}}}-\varepsilon\left(x_{i+\frac{1}{2}}\right) \widetilde{\left(v g_{h}\right)_{i+\frac{1}{2}}} \phi_{i}^{k}\left(x_{i+\frac{1}{2}}^{-}\right)\right. \\
& \left.+\varepsilon\left(x_{i-\frac{1}{2}}\right) \widetilde{\left(v g_{h}\right)_{i-\frac{1}{2}}} \phi_{i}^{k}\left(x_{i-\frac{1}{2}}^{+}\right)\right)-\omega_{k} h_{i} g_{i}^{k}+A_{i}^{k} \omega_{k} h_{i} r_{i}^{k} M_{i}^{k} / \sqrt{T_{i}^{k}} \\
& \omega_{k} h_{i} r_{i}^{k}=-\left.\sum_{k^{\prime}=0}^{K} \omega_{k^{\prime}} h_{i} T_{i}^{k^{\prime}} \frac{d \phi_{i}^{k}(x)}{d x}\right|_{x=x_{i}^{k^{\prime}}}+\widehat{T}_{h, i+\frac{1}{2}} \phi_{i}^{k}\left(x_{i+\frac{1}{2}}^{-}\right)-\widehat{T}_{h, i-\frac{1}{2}} \phi_{i}^{k}\left(x_{i-\frac{1}{2}}^{+}\right) .
\end{aligned}
$$

Here $M_{i}^{k^{\prime}}=\left.M_{h}\right|_{x=x_{i}^{k^{\prime}}}, \Pi_{i}^{k}=\Pi_{M_{i}^{k}}$ and $r_{i}^{k}=\left.r_{h}\right|_{x=x_{i}^{k}}$. And the nodal values of $T_{i}^{k}=\left.T_{h}\right|_{x=x_{i}^{k}}$ and $A_{i}^{k}=\left.A_{h}\right|_{x=x_{i}^{k}}$ are obtained from $U_{i}^{k}$ based on (2.3) and (2.10).

To the end, we still need to discretize the $v$-direction. In this work, $\Omega_{v}=\left[-V_{c}, V_{c}\right]$ is discretized uniformly with $N_{v}$ points, $\left\{v_{j}\right\}_{j=1}^{N_{v}}$. For the integration in $v$, the mid-point rule is applied, which is spectrally accurate for smooth functions with periodic boundary conditions or with a compact support [13], as there is no differential operator acting on $v$. However, this discretization in $v$ does not guarantee the property of $\langle v m g\rangle=0$ at the discrete level, also see in [32], yet in [40] we have found it sufficiently accurate for all test cases that we have performed.

\subsection{IMEX time discretization}

Now we will formulate the IMEX RK time discretizations for the semi-discrete schemes introduced in Section 3.1. First we rewrite the scheme in a compact form as follows. Find $U_{h}(\cdot, t) \in \mathbf{Z}_{h}^{K}, g_{h}(\cdot, v, t), r_{h}(\cdot, t) \in Z_{h}^{K}$, such that $\forall \phi, \psi, \varphi \in Z_{h}^{K}$ and $\forall i$,

$$
\begin{aligned}
\left(\partial_{t} U_{h}, \phi\right)+F_{h}\left(U_{h}, \phi\right) & =D_{h}\left(\varepsilon(x) g_{h}, \phi\right) \\
\left(\varepsilon(x) \partial_{t} g_{h}, \psi\right)+b_{h, v}\left(\varepsilon(x) g_{h}, \psi\right) & =s_{h}^{(1)}\left(g_{h}, \psi\right)+s_{h, v}^{(2)}\left(U_{h}, r_{h}, \psi\right) \\
\left(r_{h}, \varphi\right)=H_{h}\left(U_{h}, \varphi\right) &
\end{aligned}
$$


where

$$
\begin{aligned}
F_{h}\left(U_{h}, \phi\right) & =-\int_{\Omega_{x}} F\left(U_{h}\right) \frac{d \phi(x)}{d x} d x-\sum_{i} \hat{F}_{h, i+\frac{1}{2}}[\phi]_{i+\frac{1}{2}}, \\
D_{h}\left(\varepsilon(x) g_{h}, \phi\right) & \left.=\int_{\Omega_{x}} \varepsilon(x)\left\langle v m g_{h}\right\rangle \frac{d \phi(x)}{d x} d x+\sum_{i} \varepsilon\left(x_{i+\frac{1}{2}}\right) \widehat{\left\langle v m g_{h}\right.}\right\rangle_{i+\frac{1}{2}}[\phi]_{i+\frac{1}{2}}, \\
b_{h, v}\left(\varepsilon(x) g_{h}, \psi\right) & =\int_{\Omega_{x}}\left(\mathbf{I}-\Pi_{M_{h}}\right) \mathcal{D}_{h, 1}\left(\varepsilon(x) v g_{h}\right) \psi d x, \\
s_{h}^{(1)}\left(g_{h}, \psi\right) & =-\int_{\Omega_{x}} g_{h} \psi d x, \quad s_{h, v}^{(2)}\left(U_{h}, r_{h}, \psi\right)=-\int_{\Omega_{x}} A_{h} \frac{r_{h}}{\sqrt{T_{h}}} M_{h} \psi d x, \\
H_{h}\left(U_{h}, \varphi\right) & =-\left.\left(\int_{\Omega_{x}} T_{h} \frac{d \varphi}{d x} d x+\sum_{i} \hat{T}_{h, i+\frac{1}{2}}[\varphi]_{i+\frac{1}{2}}\right)\right|_{T_{h}=T_{h}\left(U_{h}\right)} .
\end{aligned}
$$

High order globally stiffly accurate IMEX schemes can be characterized by a double Butcher Tableau

$$
\begin{array}{l|ll|l}
\tilde{c} & \tilde{A} \\
\hline & \tilde{b}^{\top}
\end{array} \quad \begin{array}{c|c}
c & A \\
\hline & b^{\top}
\end{array},
$$

where $\tilde{A}=\left(\tilde{a}_{i j}\right)$ is an $s \times s$ lower triangular matrix with zero diagonal for an explicit scheme, and $A=\left(a_{i j}\right)$ is an $s \times s$ lower triangular matrix with the diagonal entries not all being zero for a diagonally implicit RK (DIRK) method. The coefficients $\tilde{c}$ and $c$ are given by the standard relations

$$
\tilde{c}_{i}=\sum_{j=1}^{i-1} \tilde{a}_{i j}, \quad c_{i}=\sum_{j=1}^{i} a_{i j}
$$

and vectors $\tilde{b}=\left(\tilde{b}_{j}\right)$ and $b=\left(b_{j}\right)$ represent the quadrature weights for internal stages of the RK method. The IMEX RK scheme is defined to be globally stiffly accurate if $\tilde{c}_{s}=c_{s}=1$ and $a_{s j}=b_{j}, \tilde{a}_{s j}=\tilde{b}_{j}, \forall j=1, \cdots, s$.

Now the fully-discrete scheme using the Butcher notation can be written as follows. Given $U_{h}^{n} \in \mathbf{Z}_{h}^{K}$ and $g_{h}^{n} \in Z_{h}^{K}$, we look for $U_{h}^{n+1} \in \mathbf{Z}_{h}^{K}$ and $g_{h}^{n+1} \in Z_{h}^{K}$, such that $\forall \phi, \psi \in Z_{h}^{K}$,

$$
\begin{aligned}
\left(U_{h}^{n+1}, \phi\right) & =\left(U_{h}^{n}, \phi\right)-\Delta t \sum_{l=1}^{s} \tilde{b}_{l}\left(F_{h}\left(U_{h}^{(l)}, \phi\right)-D_{h}\left(\varepsilon(x) g_{h}^{(l)}, \phi\right)\right), \\
\left(\varepsilon(x) g_{h}^{n+1}, \psi\right) & =\left(\varepsilon(x) g_{h}^{n}, \psi\right)-\Delta t \sum_{l=1}^{s} \tilde{b}_{l} b_{h, v}\left(\varepsilon(x) g_{h}^{(l)}, \psi\right)+\Delta t \sum_{l=1}^{s} b_{l}\left(s_{h}^{(1)}\left(g_{h}^{(l)}, \psi\right)+s_{h, v}^{(2)}\left(U_{h}^{(l)}, r_{h}^{(l)}, \psi\right)\right) .
\end{aligned}
$$

Here the approximations at the internal stages of one RK step, $U_{h}^{(l)} \in \mathbf{Z}_{h}^{K}$ and $g_{h}^{(l)}, r_{h}^{(l)} \in Z_{h}^{K}$ 
with $l=1, \cdots, s$, satisfy

$$
\begin{aligned}
\left(U_{h}^{(l)}, \phi\right) & =\left(U_{h}^{n}, \phi\right)-\Delta t \sum_{j=1}^{l-1} \tilde{a}_{l j}\left(F_{h}\left(U_{h}^{(j)}, \phi\right)-D_{h}\left(\varepsilon(x) g_{h}^{(j)}, \phi\right)\right) \\
\left(\varepsilon(x) g_{h}^{(l)}, \psi\right) & =\left(\varepsilon(x) g_{h}^{n}, \psi\right)-\Delta t \sum_{j=1}^{l-1} \tilde{a}_{l j} b_{h, v}\left(\varepsilon(x) g_{h}^{(j)}, \psi\right)+\Delta t \sum_{j=1}^{l} a_{l j}\left(s_{h}^{(1)}\left(g_{h}^{(j)}, \psi\right)+s_{h, v}^{(2)}\left(U_{h}^{(j)}, r_{h}^{(j)}, \psi\right)\right) \\
\left(r_{h}^{(l)}, \varphi\right) & =H_{h}\left(U_{h}^{(l)}, \varphi\right)
\end{aligned}
$$

for any $\phi, \psi, \varphi \in Z_{h}^{K}$. One can solve the IMEX scheme in a stage-by-stage fashion for $l=$ $1, \cdots, s$, that is, we first solve $U_{h}^{(l)}$ explicitly from the equation $(3.15 \mathrm{a})$, then plug $U_{h}^{(l)}$ into $(3.15 \mathrm{c})$ to solve $r_{h}^{(l)}$, and finally solve $g_{h}^{(l)}$ from $(3.15 \mathrm{~b})$.

The third order IMEX scheme we used in our simulations is the globally stiffly accurate $\operatorname{ARS}(4,4,3)$ scheme [3] with a double Butcher Tableau

\begin{tabular}{c|cccccc|ccccc}
0 & 0 & 0 & 0 & 0 & 0 & 0 & 0 & 0 & 0 & 0 & 0 \\
$1 / 2$ & $1 / 2$ & 0 & 0 & 0 & 0 & $1 / 2$ & 0 & $1 / 2$ & 0 & 0 & 0 \\
$2 / 3$ & $11 / 18$ & $1 / 18$ & 0 & 0 & 0 & $2 / 3$ & 0 & $1 / 6$ & $1 / 2$ & 0 & 0 \\
$1 / 2$ & $5 / 6$ & $-5 / 6$ & $1 / 2$ & 0 & 0 & $1 / 2$ & 0 & $-1 / 2$ & $1 / 2$ & $1 / 2$ & 0 \\
1 & $1 / 4$ & $7 / 4$ & $3 / 4$ & $-7 / 4$ & 0 & 1 & 0 & $3 / 2$ & $-3 / 2$ & $1 / 2$ & $1 / 2$ \\
\hline & $1 / 4$ & $7 / 4$ & $3 / 4$ & $-7 / 4$ & 0
\end{tabular}

For the fully discrete DG-IMEX scheme (3.14)-(3.15), with operators specified in (3.11), the Proposition 3.4 in [40] has shown that, for $0<\varepsilon \ll 1$, the scheme is asymptotically equivalent, up to $\mathcal{O}\left(\varepsilon^{2}\right)$, to a local DG (LDG) method in its nodal form for the compressible Navier-Stokes equations

$$
\partial_{t}\left(\begin{array}{c}
\rho \\
\rho u \\
E
\end{array}\right)+\partial_{x}\left(\begin{array}{c}
\rho u \\
\rho u^{2}+p I \\
(E+p) u
\end{array}\right)=\varepsilon \partial_{x}\left(\begin{array}{c}
0 \\
0 \\
\frac{3}{2} \rho T \partial_{x} T
\end{array}\right),
$$

and the LDG scheme evolved in time by an explicit RK method characterized by a Butcher table $\tilde{A}, \tilde{b}$ and $\tilde{c}$ in (3.12) is defined as follows: find $U_{h}^{n+1}(\cdot, t), U_{h}^{(l)} \in \mathbf{Z}_{h}^{K}$ and $r_{h}^{(l)}(\cdot, t) \in Z_{h}^{K}$ with $l=1, \cdots, s$, such that $\forall \phi, \varphi \in Z_{h}^{K}$,

$$
\left(U_{h}^{n+1}, \phi\right)=\left(U_{h}^{n}, \phi\right)-\Delta t \sum_{l=1}^{s} \tilde{b}_{l}\left(F_{h}\left(U_{h}^{(l)}, \phi\right)-\varepsilon F_{h}^{(v i s)}\left(U_{h}^{(l)}, r_{h}^{(l)}, \phi\right)\right)
$$

with

$$
\left(U_{h}^{(l)}, \phi\right)=\left(U_{h}^{n}, \phi\right)-\Delta t \sum_{j=1}^{l-1} \tilde{a}_{l j}\left(F_{h}\left(U_{h}^{(j)}, \phi\right)-\varepsilon F_{h}^{(v i s)}\left(U_{h}^{(j)}, r_{h}^{(j)}, \phi\right)\right)
$$

and

$$
\left(r_{h}^{(l)}, \varphi\right)=-\sum_{i}\left(\int_{I_{i}} T_{h}^{(l)} \frac{d \varphi}{d x} d x+\widehat{T}_{h, i+\frac{1}{2}}^{(l)}[\varphi]_{i+\frac{1}{2}}\right) .
$$

Its nodal form can be similarly defined as in Section 3.1 and is omitted for brevity. When $\varepsilon=0$, if we omit the $\varepsilon$ terms in (3.18) and (3.19), it becomes a RK DG scheme for the compressible Euler system. 


\subsection{Regime indicators}

In this section, we follow [27] to introduce the regime indicators but group all the computational cells into three classes: (I) Euler regime, (II) Navier-Stokes (NS) regime, (III) kinetic regime. We start all computational cells in the kinetic regime unless being indicated otherwise. We have the criteria for both directions: from/to kinetic to/from hydrodynamic regimes. In particular, we use macroscopic quantities to determine when the hydrodynamic description breaks down, and use microscopic ones to determine when the kinetic description is not necessary and a hydrodynamic description would be sufficient. Moreover, we also distinguish between compressible Euler and Navier-Stokes in the hydrodynamics regime, where a finer criteria is proposed.

For simplicity, we consider the problem in one dimensional case. As in [27], we use the following short hand notation for the rescaled microscopic velocity $V(v)=\frac{v-u}{\sqrt{T}}$, then $A$ and $B$ defined in (2.10) can be written as $A=0$ and $B(V)=\frac{1}{2}\left(V^{2}-3\right) V$. We let $\bar{B}:=\frac{1}{\rho} \int_{\mathbb{R}} B(V) f(v) d v$.

\subsubsection{From fluid to kinetic: The moment realizability criterion}

Following [30, 27], we define the moment realizability matrix as

$$
\mathbf{M}:=\frac{1}{\rho} \int_{\mathbb{R}} \mathbf{m} \otimes \mathbf{m} f(v) d v
$$

where $\mathbf{m}$ is the collisional invariant vector for $V=(v-u) / \sqrt{T}$,

$$
\mathbf{m}:=\left(1, V, \frac{1}{\sqrt{2}}\left(V^{2}-1\right)\right) .
$$

From the properties of the moments defined in (2.3), we have

$$
\begin{aligned}
\mathbf{M} & =\frac{1}{\rho} \int_{\mathbb{R}}\left(\begin{array}{ccc}
1 & V & \frac{1}{\sqrt{2}}\left(V^{2}-1\right) \\
V & V^{2} & \frac{1}{\sqrt{2}}\left(V^{2}-1\right) V \\
\frac{1}{\sqrt{2}}\left(V^{2}-1\right) & \frac{1}{\sqrt{2}}\left(V^{2}-1\right) V & \frac{1}{2}\left(V^{2}-1\right)^{2}
\end{array}\right) f(v) d v \\
& =\left(\begin{array}{ccc}
1 & 0 & 0 \\
0 & 1 & \sqrt{2} \bar{B} \\
0 & \sqrt{2} \bar{B} & \bar{C}
\end{array}\right)
\end{aligned}
$$

where $\bar{C}$ is the dimensionless fourth order moment of $f$ :

$$
\bar{C}=\frac{1}{2 \rho} \int_{\mathbb{R}}\left(V^{2}-1\right)^{2} f(v) d v
$$

Now let us consider different orders for the approximation of $f$ with respect to $\varepsilon$. The values of $\bar{B}$ and $\bar{C}$ can then be explicitly determined.

- Zeroth order: Compressible Euler system.

We have $f=M_{U}$ and $\bar{B}=0$ since it only involves odd, centered moments of $f$. Direct computation shows that $\bar{C}=1$. In this case, $\mathbf{M}$ is the identity matrix, all the eigenvalues are 1 , we denote it as $\nu_{\text {Euler }}=1$. 
- First order: Compressible Navier-Stokes system.

We have $f=M_{U}+\varepsilon g=M_{U}-\varepsilon A \frac{T_{x}}{\sqrt{T}} M$, with analytical expression for the projection term defined in (2.9). And we can find that $\bar{B}=-\varepsilon \frac{\kappa}{\rho T^{3 / 2}} T_{x}$, where $q=\kappa T_{x}$ is the heat flux. For $\bar{C}$, using symmetry arguments, we still have $\bar{C}=1$. For the matrix $\mathbf{M}$, beside one eigenvalue to be 1 , the other two eigenvalues would be $1 \pm \sqrt{2} \bar{B}$. We denote the largest absolute eigenvalue for the compressible Navier-Stokes equations as $\nu_{N S}=1+\sqrt{2}|\bar{B}|=1+\varepsilon \frac{\sqrt{2} \kappa}{\rho T^{3 / 2}}\left|T_{x}\right|$.

- Second order: Burnett equations.

The second order Burnett equations will be used as a reference for the kinetic equation, when the fluid is far away from the thermal equilibrium. It would be complicated to derive the explicit expression for $\bar{B}$ under the micro-macro decomposition framework up to second order. Instead, we directly use the one obtained from Chapman-Enskog expansion as in [27] (eq. (16)). Since $D(u)=0$, here the expression for $\bar{B}$ would be

$$
\bar{B}=-\varepsilon \frac{\kappa}{\rho T^{3 / 2}} T_{x}-\varepsilon^{2} \frac{\mu^{2}}{\sqrt{T}}\left(\frac{25}{6} u_{x} T_{x}-\frac{5}{3}\left(T u_{x x}+7 u_{x} T_{x}\right)\right) .
$$

Similarly, beside 1 is one eigenvalue of $\mathbf{M}$, the other two eigenvalues are $1+\frac{1}{2}(\bar{C}-1 \pm$ $\left.\sqrt{(\bar{C}-1)^{2}+8 \bar{B}^{2}}\right)$, so that we denote $\nu_{B}=1+\frac{1}{2}\left(|\bar{C}-1|+\sqrt{(\bar{C}-1)^{2}+8 \bar{B}^{2}}\right)$ with $\bar{B}$ in (3.25) and $\bar{C}$ in (3.24).

A criterion to determine whether a hydrodynamic description breaks down is to find out the deviation of the eigenvalues in the corresponding fluid models away from the reference eigenvalue which we denoted as $\nu_{B}$. We propose a criterion with the following two steps:

- Step 1: If a cell is in the Euler regime, it will be classified as the NS regime if $\mid \nu_{B}-$ $\nu_{\text {Euler }}||_{x_{i}}>\eta_{0}$.

- Step 2: For all the cells in the NS regime (including those from the Euler regime in Step 1), if $\left.\left|\nu_{B}-\nu_{N S}\right|\right|_{x_{i}}>\eta_{1}$, they will be classified as the kinetic regime.

The best choice of the thresholds $\eta_{0}$ and $\eta_{1}$ is problem dependent. In our numerical tests, we all take $\eta_{0}=10^{-2}$ and $\eta_{1}=10^{-1}$. In order to avoid classifying a smooth extremum (where $T_{x}$ and $u_{x}$ might both be zero) as the Euler regime (which may not be physically accurate), we propose to change a cell from the Euler regime into the NS regime if both of its neighbors are in the NS regime.

Remark 3.1. In our numerics, the first and second derivatives, such as $T_{x}, u_{x}$ and $u_{x x}$ when computing the eigenvalues, are approximated by a DG or LDG discretization with central fluxes. For example, letting $r_{h}$ approximate $T_{x}$, the DG formulation to obtain $r_{h}$ is the same as in (3.7) with $\widehat{T}=\left(T^{+}+T^{-}\right) / 2$, similarly for $u_{x}$. Letting $\zeta_{h}$ and $\eta_{h}$ approximate $u_{x}$ and $u_{x x}$ respectively, an LDG formulation to obtain $\eta_{h}$ is as follows: for any basis functions $\phi$ and $\psi$ in $I_{i}$ as in (3.8),

$$
\begin{aligned}
\int_{I_{i}} \eta_{h} \phi d x & =-\int_{I_{i}} \zeta_{h} \frac{d \phi}{d x} d x+\widehat{\zeta}_{h, i+\frac{1}{2}} \phi_{i+\frac{1}{2}}^{-}-\widehat{\zeta}_{h, i-\frac{1}{2}} \phi_{i-\frac{1}{2}}^{+}, \\
\int_{I_{i}} \zeta_{h} \psi d x & =-\int_{I_{i}} u_{h} \frac{d \psi}{d x} d x+\widehat{u}_{h, i+\frac{1}{2}} \psi_{i+\frac{1}{2}}^{-}-\widehat{u}_{h, i-\frac{1}{2}} \psi_{i-\frac{1}{2}}^{+},
\end{aligned}
$$


with $\widehat{\zeta}=\left(\zeta^{+}+\zeta^{-}\right) / 2$ and $\widehat{u}=\left(u^{+}+u^{-}\right) / 2 . u_{h}$ is the numerical velocity approximating $u$.

\subsubsection{From kinetic to fluid}

The criterion in this direction would simply be a comparison between the kinetic density $f=$ $M+\varepsilon g$ and the truncated distribution $f_{k}$ whose moments match those of $f$ and $k$ is the order of the macroscopic model. In particular, a kinetic description will be changed to a hydrodynamic closure of $k$ if

$$
\left\|f(t, x, \cdot)-f_{k}(t, x, \cdot)\right\|_{L_{M}^{2}} \leq \delta_{0}
$$

with a weighted $L^{2}$ norm defined as $\|\cdot\|_{L_{M}^{2}}=\left(\int_{\mathbb{R}}|\cdot|^{2} / M_{U} d v / \rho\right)^{1 / 2}$. For example, $f_{1}(t, x, v)=$ $M$ and $\left\|f(t, x, v)-f_{1}(t, x, v)\right\|_{L_{M}^{2}}=\varepsilon\|g(t, x, v)\|_{L_{M}^{2}}$, while $f_{2}(t, x, v)=M-\varepsilon A \frac{T_{x}}{\sqrt{T}} M$ and $\left\|f(t, x, v)-f_{2}(t, x, v)\right\|_{L_{M}^{2}}=\varepsilon\left\|g(t, x, v)+A \frac{T_{x}}{\sqrt{T}} M\right\|_{L_{M}^{2}}$. The criterion is implemented in the following two steps:

- Step 1: For a cell not in the Euler regime (including both NS and kinetic), if $\varepsilon\|g(t, x, v)\|_{L_{M}^{2}}<$ $\delta_{0}$, it is added to the Euler regime.

- Step 2: Otherwise, if it is in the kinetic regime and $\varepsilon\left\|g(t, x, v)+A \frac{T_{x}}{\sqrt{T}} M\right\|_{L_{M}^{2}}<\delta_{0}$, it is added to the NS regime.

In our numerical tests, we take $\delta_{0}=10^{-3}$.

\subsection{Interface coupling conditions and algorithm flow chart}

Thanks to the micro-macro formulation, in all of our computational cells, the macroscopic variables $U$ are solved; while the microscopic variable $g$ is updated only in kinetic cells. Therefore, the only interface coupling condition we need to impose is from a fluid to a kinetic cell, where the boundary condition of $g$ is needed from the fluid cell. It is indeed very natural to do so by letting $g=-A \frac{T_{x}}{\sqrt{T}} M$. Due to the compactness of the DG property, such boundary condition is needed only for the cell directly adjacent to the interface and no buffer zone is needed.

Our hierarchical algorithm with regime indicators realizes an adaptive seamless coupling between hydrodynamic and kinetic solvers at different levels. The scheme can be briefly sketched as follows:

- Initially at $T=0$, all cells are classified in kinetic regime unless being indicated otherwise. We apply the criteria in Section 3.3 to classify the cells into NS or Euler regimes.

- From $t^{n}$ to $t^{n+1}$ :

- For Euler, NS and kinetic regions, we apply the corresponding RK DG, RK LDG and NDG-IMEX solvers as described in Section 3.1 and Section 3.2. In all computational cells, macroscopic information $U$ is stored and being updated, while the microscopic components $g$ is stored and computed only in kinetic regimes. At boundary elements along the interface between hydrodynamic (Euler or NS) and kinetic cells, we let $g=$ $-A \frac{T_{x}}{\sqrt{T}} M$ as the boundary condition for microscopic component in kinetic regimes as can be seen from (2.8) and (3.2). 
- At the final stage of a multi-stage Runge-Kutta method, the criteria specified in Section 3.3 are applied to dynamically update the kinetic, NS and Euler regions.

We again emphasize that the seamless coupling of different solvers are due to the compactness property of the DG method and the asymptotic equivalence of the kinetic solver with macroscopic ones. In particular, the NDG-IMEX scheme for the kinetic equation automatically becomes a RK DG scheme by assuming the microscopic quantity $\varepsilon g=0$ and it automatically becomes a RK LDG scheme for CNS system by assuming $g=-A \frac{T_{x}}{\sqrt{T}} M$. The macroscopic solvers avoid updating the high-dimensional microscopic variable $g$, leading to significant computational savings.

\section{Numerical Examples}

In this section, we will apply the regime indicators to the NDG-IMEX scheme for the micromacro decomposed BGK equation (2.6) with constant $\varepsilon$, and (2.7) with variable $\varepsilon(x)$. We take the third order NDG-IMEX scheme with 3-point Gauss quadrature, corresponding to a Lagrangian polynomial basis of degree 2. The corresponding DG and LDG schemes in the compressible Euler and Navier-Stokes regimes are also third order. The time step is chosen as $\Delta t=C F L h / \max \left(\Lambda, V_{c}\right)$, where $C F L=0.05$ and $\Lambda=\||u|+\sqrt{\gamma T}\|_{\infty}$ is the maximal absolute eigenvalue of $\partial F(U) / \partial U$ over the spatial domain. The velocity domain $\Omega=\left[-V_{c}, V_{c}\right]$ is set to be large enough. The TVB (total-variation-bounded) limiter [35] with the parameter $M_{t v b}=1$ is used and is only applied on $U_{h}$.

We will consider three different hierarchical schemes, where the hydrodynamic regime may contain only the Euler regime, the NS regime, or both the Euler and NS regimes, which we will denote as Euler-Kinetic, NS-Kinetic and Euler-NS-Kinetic schemes, respectively. The NS reference solutions are computed by solving the compressible NS equations (3.17) with (3.18) (3.20), while the Euler reference solutions are just omitting the two $\varepsilon$ terms in (3.18) - (3.19), which are denoted as solid lines in the following figures. The kinetic reference solutions are computed by solving (2.7) with (3.14) and (3.15), which are denoted as dashed lines in the following figures. For all examples in this section, the numerical solutions and the reference solutions are computed with 50 and 200 cells, respectively.

\subsection{Sod problem}

First we consider the sod shock tube problem with initial conditions to be

$$
(\rho, u, T)= \begin{cases}(1,0,1) & \text { if } x<0.5 \\ (0.125,0,0.8) & \text { if } x>0.5\end{cases}
$$

on the domain $[-0.2,1.2], v \in[-4.5,4.5]$.

We report the results for $\varepsilon=10^{-2}$ in Figures 4.1-4.4 and for $\varepsilon=10^{-3}$ in Figures 4.5-4.8, for Euler-Kinetic, NS-Kinetic and Euler-NS-Kinetic schemes, respectively. It is observed that in regions where the hydrodynamic and kinetic solutions differ from each other, the kinetic solver is turned on and the solutions approximate the kinetic reference solutions well. The results for all three cases match each other very well. The NS-Kinetic scheme has less kinetic cells than 
the Euler-Kinetic scheme. The Euler-NS-Kinetic scheme with all three regimes together shows that NS regime well connects the Euler and kinetic regimes.

For this problem, we also compare the computational cost for different indicators, as well as the full kinetic scheme. In Table 4.1, we can see that the Euler-NS-Kinetic scheme can save $72 \%$ for $\varepsilon=10^{-2}$ and $87 \%$ for $\varepsilon=10^{-3}$ as compared to the full kinetic scheme, while the NSKinetic scheme saves $74 \%$ for $\varepsilon=10^{-2}$ and $85 \%$ for $\varepsilon=10^{-3}$. The Euler-Kinetic scheme has more kinetic cells than the other two hierarchical schemes and it costs the most, similarly for the following examples. However, for this example, it still saves $55 \%$ for $\varepsilon=10^{-2}$ and $70 \%$ for $\varepsilon=10^{-3}$. This is due to that the kinetic and Euler reference solutions are very close, especially when $\varepsilon=10^{-3}$, the regime indicators are very active for this problem. The Euler-NS-Kinetic scheme performs almost the same as the NS-Kinetic one, costing a little higher for $\varepsilon=10^{-2}$ and a little lower for $\varepsilon=10^{-3}$.

Table 4.1: Comparison of the computational time (seconds) for different indicators. Sod problem.

\begin{tabular}{|c|c|c|c|c|}
\hline method & Euler-NS-Kinetic & NS-Kinetic & Euler-Kinetic & Full kinetic \\
\hline$\varepsilon=10^{-2}$ & 8.38 & 7.81 & 13.40 & 30.19 \\
\hline$\varepsilon=10^{-3}$ & 3.78 & 4.28 & 8.28 & 28.57 \\
\hline
\end{tabular}

\subsection{Blast wave problem}

For the blast wave problem, the initial condition is given by

$$
(\rho, u, T)= \begin{cases}(1,1,2) & \text { if } x<0.2 \\ (1,0,0.25) & \text { if } 0.2 \leq x \leq 0.8 \\ (1,-1,2) & \text { if } x>0.8\end{cases}
$$

with reflective boundary condition in the $x$ direction on the domain $[0,1]$ and $v \in[-9,9]$.

Similarly, we report the results for $\varepsilon=10^{-2}$ and $\varepsilon=10^{-3}$ for Euler-Kinetic, NS-Kinetic and Euler-NS-Kinetic schemes in Figures 4.9-4.12 and 4.13-4.16, respectively. For this problem, when $\varepsilon=10^{-2}$, the kinetic reference solution deviates slightly away from the fluid reference solutions, and most computational cells are assigned into the kinetic regime. However, when $\varepsilon$ becomes smaller, that is when $\varepsilon=10^{-3}$, the kinetic reference solution is getting close to the fluid reference solutions. It can be observed from Figure 4.13 and Figure 4.14 that the Euler and NS solvers are turned on in larger regions, leading to computational savings. If one compares Figure 4.13-4.15, "best" solvers that well balance computational effectiveness (in capturing reference solutions) and efficiency (in saving computational time) are adaptively chosen by the criteria.

Similar to the Sod problem, we compare the computational cost for the blast wave problem in Table 4.2. As we can see, when $\varepsilon=10^{-2}$, most of the cells are identified in the kinetic regime. The Euler-NS-Kinetic and NS-Kinetic schemes only save around $10 \% \sim 15 \%$, while the Euler-Kinetic scheme takes even larger computational time than the full kinetic scheme. Also the computational time for the Euler-NS-Kinetic scheme is slightly larger than that of the 

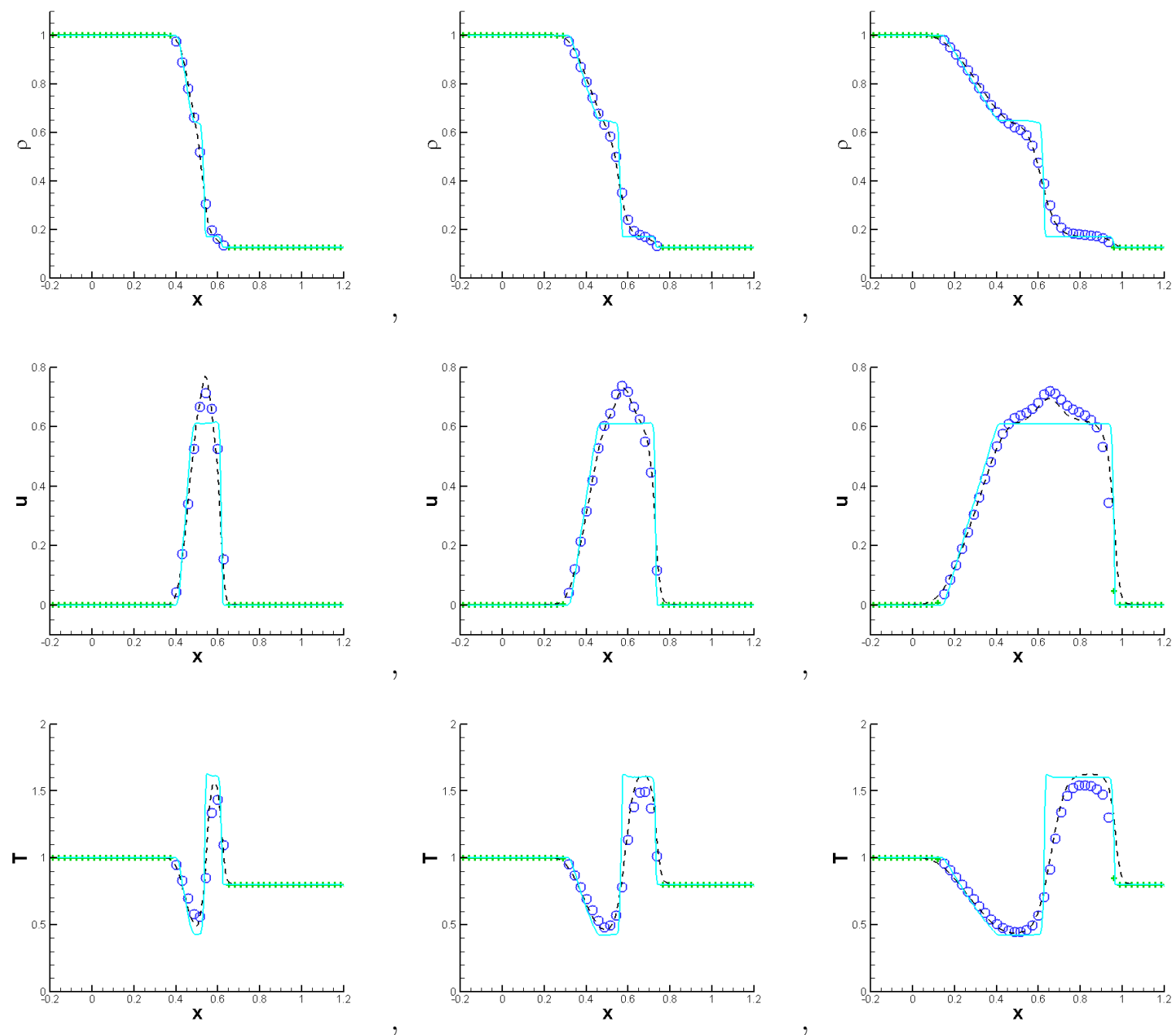

Figure 4.1: Sod problem on the domain $[-0.2,1.2] \times[-4.5,4.5]$. Symbols: $N_{x}=50$ and $N_{v}=100$ with NDG3. From left to right: time $t=0.05,0.1,0.2$. From top to bottom, the density $\rho$, the mean velocity $u$ and the temperature $T$. Symbols: ' + ' is Euler, circle is kinetic. Solid line: the Euler reference solution. Dashed line: the kinetic reference solution. $\varepsilon=10^{-2}$, Euler-Kinetic.

NS-Kinetic one. This is due to that computing the regime indicators takes extra computational time. When $\varepsilon=10^{-3}$, the solution becomes closer to the NS reference solution. The EulerNS-Kinetic and NS-Kinetic schemes can save up to $80 \%$, while the Euler-Kinetic one can save around $60 \%$. Also the Euler-NS-Kinetic scheme takes larger time than the NS-Kinetic one. For the 1D problem, the compressible NS equations (3.17) have only one extra term than the compressible Euler equations. We might have taken slightly larger time to compute the regime indicators and the logic decisions for the Euler-NS-Kinetic scheme, as compared to compute the extra term than the NS-Kinetic scheme. For high dimensional problems, as we can see from (2.9), the projection involves much more terms. Hence the savings of the Euler-NS-Kinetic scheme would become more significant. 

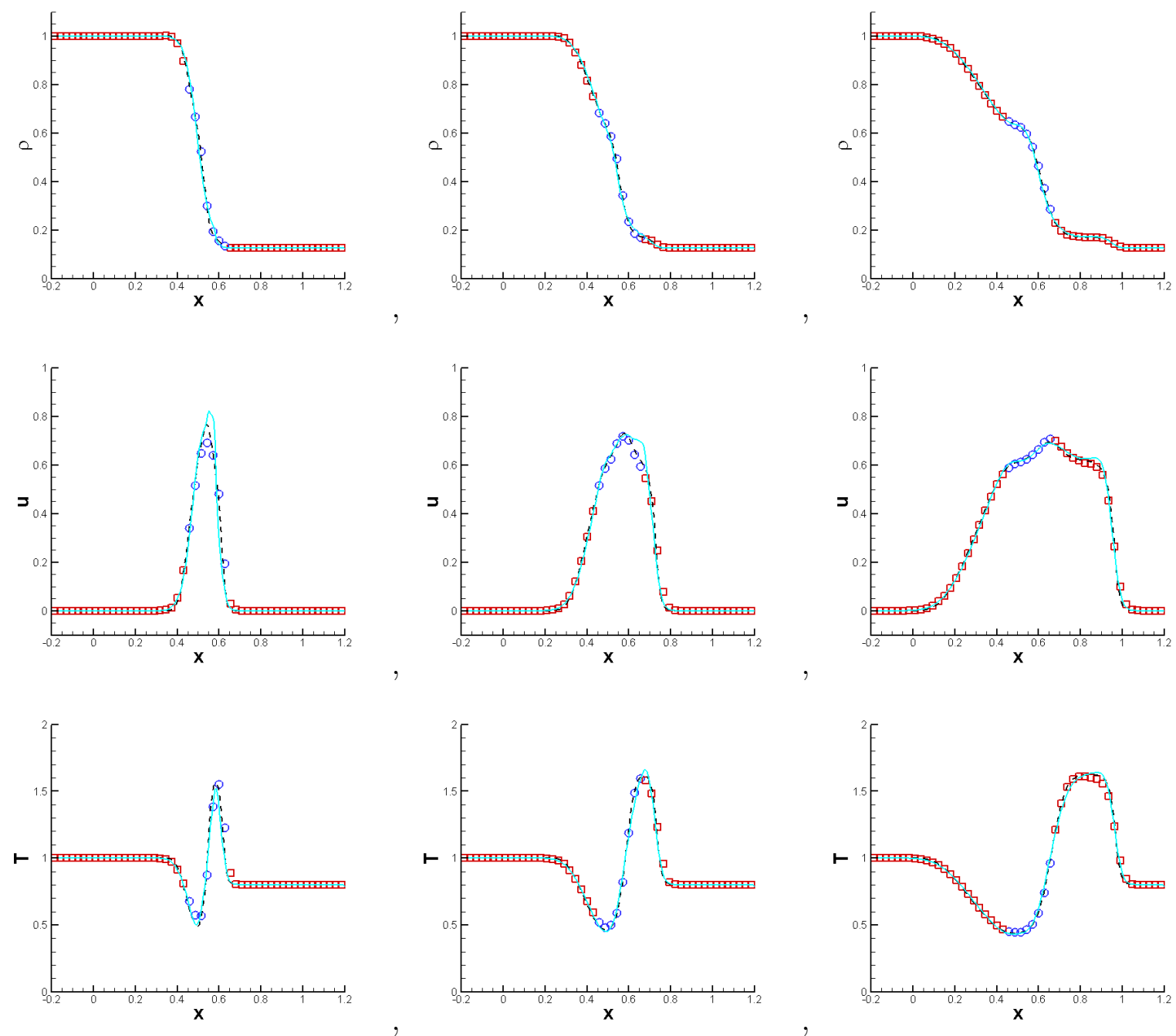

Figure 4.2: Sod problem on the domain $[-0.2,1.2] \times[-4.5,4.5]$. Symbols: $N_{x}=50$ and $N_{v}=100$ with NDG3. From left to right: time $t=0.05,0.1,0.2$. From top to bottom, the density $\rho$, the mean velocity $u$ and the temperature $T$. Symbols: square is NS, circle is kinetic. Solid line: the NS reference solution. Dashed line: the kinetic reference solution. $\varepsilon=10^{-2}$, NS-Kinetic.

Table 4.2: Comparison of the computational cost (seconds) for different indicators. Blast wave problem.

\begin{tabular}{|c|c|c|c|c|}
\hline method & Euler-NS-Kinetic & NS-Kinetic & Euler-Kinetic & Full kinetic \\
\hline$\varepsilon=10^{-2}$ & 85.74 & 80.98 & 102.42 & 96.72 \\
\hline$\varepsilon=10^{-3}$ & 17.22 & 16.77 & 37.97 & 98.72 \\
\hline
\end{tabular}

\subsection{Mixed regime problem}

We consider an example with a variable $\varepsilon(x)$,

$$
\varepsilon(x)=\varepsilon_{0}+\frac{1}{2}\left(\tanh \left(1-a_{0} x\right)+\tanh \left(1+a_{0} x\right)\right),
$$



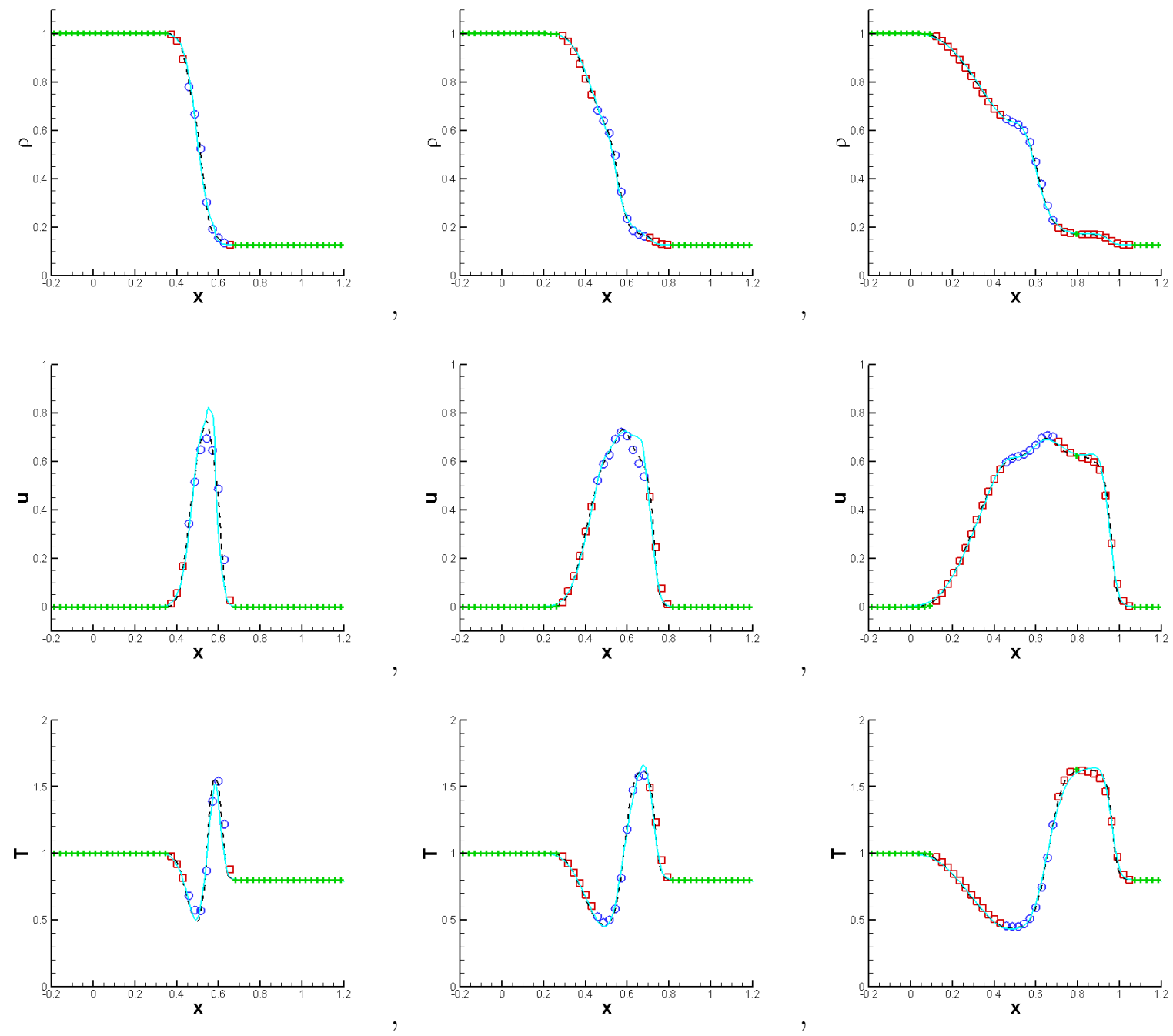

Figure 4.3: Sod problem on the domain $[-0.2,1.2] \times[-4.5,4.5]$. Symbols: $N_{x}=50$ and $N_{v}=100$ with NDG3. From left to right: time $t=0.05,0.1,0.2$. From top to bottom, the density $\rho$, the mean velocity $u$ and the temperature T. Symbols: ' + ' is Euler, square is NS, circle is kinetic. Solid line: the NS reference solution. Dashed line: the kinetic reference solution. $\varepsilon=10^{-2}$, Euler-NS-Kinetic.

The initial distribution function $f$ is far away from the Maxwellian, which is

$$
f(x, v, 0)=\frac{\tilde{\rho}}{2(2 \pi \tilde{T})^{1 / 2}}\left[\exp \left(-\frac{|v-\tilde{u}|^{2}}{2 \tilde{T}}\right)+\exp \left(-\frac{|v+\tilde{u}|^{2}}{2 \tilde{T}}\right)\right],
$$

with

$$
\tilde{\rho}(x)=1+0.875 \sin (w x), \quad \tilde{T}(x)=0.5+0.4 \sin (\omega x), \quad \tilde{u}(x)=0.75,
$$

on the spatial domain $x \in[-L, L]$, where $\omega=\pi / L$ and $L=0.5$. The initial macroscopic variables are

$$
\rho(x, 0)=\tilde{\rho}(x), \quad u(x, 0)=0, \quad T(x, 0)=\tilde{T}(x)+\tilde{u}(x)^{2},
$$



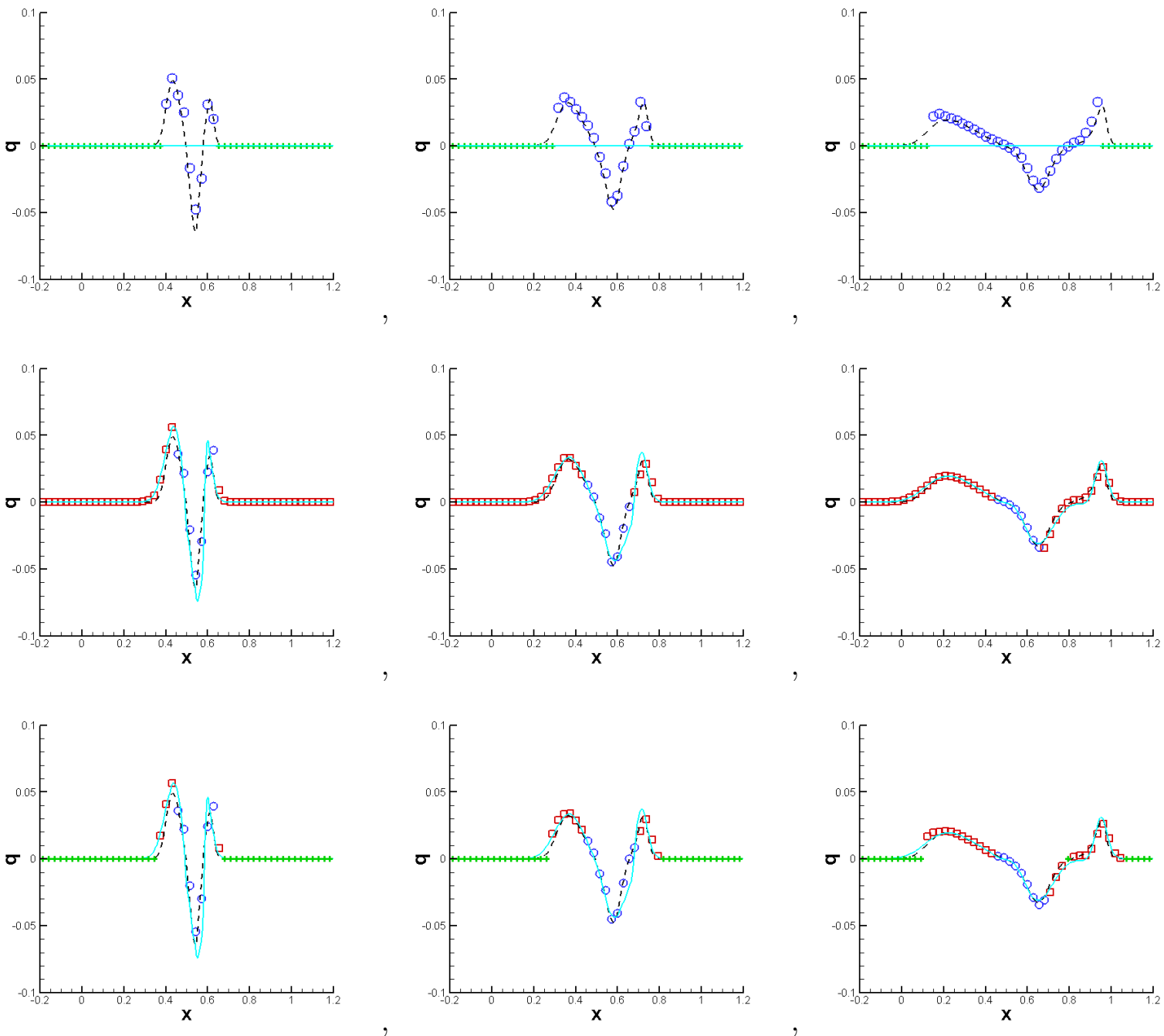

Figure 4.4: Heat flux of sod problem on the domain $[-0.2,1.2] \times[-4.5,4.5]$. Symbols: $N_{x}=50$ and $N_{v}=100$ with NDG3. From left to right: time $t=0.05,0.1,0.2$. From top to bottom, Euler-Kinetic, NS-Kinetic and Euler-NS-Kinetic. Symbols: '+' is Euler, square is NS, circle is kinetic. Solid line: the first row is the Euler reference solution, other two rows are the NS reference solution. Dashed line: the kinetic reference solution. $\varepsilon=10^{-2}$.

and the initial Maxwellian distribution is

$$
M_{U}(x, v, 0)=\frac{\rho(x, 0)}{(2 \pi T(x, 0))^{1 / 2}} \exp \left(-\frac{|v-u(x, 0)|^{2}}{2 T(x, 0)}\right) .
$$

Periodic boundary conditions are used for both $U$ and $g$ in the $x$ direction.

We first take this example with mixed regimes to test the orders of accuracy for the hierarchical Euler-NS-Kinetic scheme, which is similar to Table 4.3 in [40]. We choose $\varepsilon_{0}=10^{-6}$ and $a_{0}=11$ in $(4.1)$ and $\Omega_{v}=[-16,16]$. The $L^{1}$ errors and convergence orders for the density 

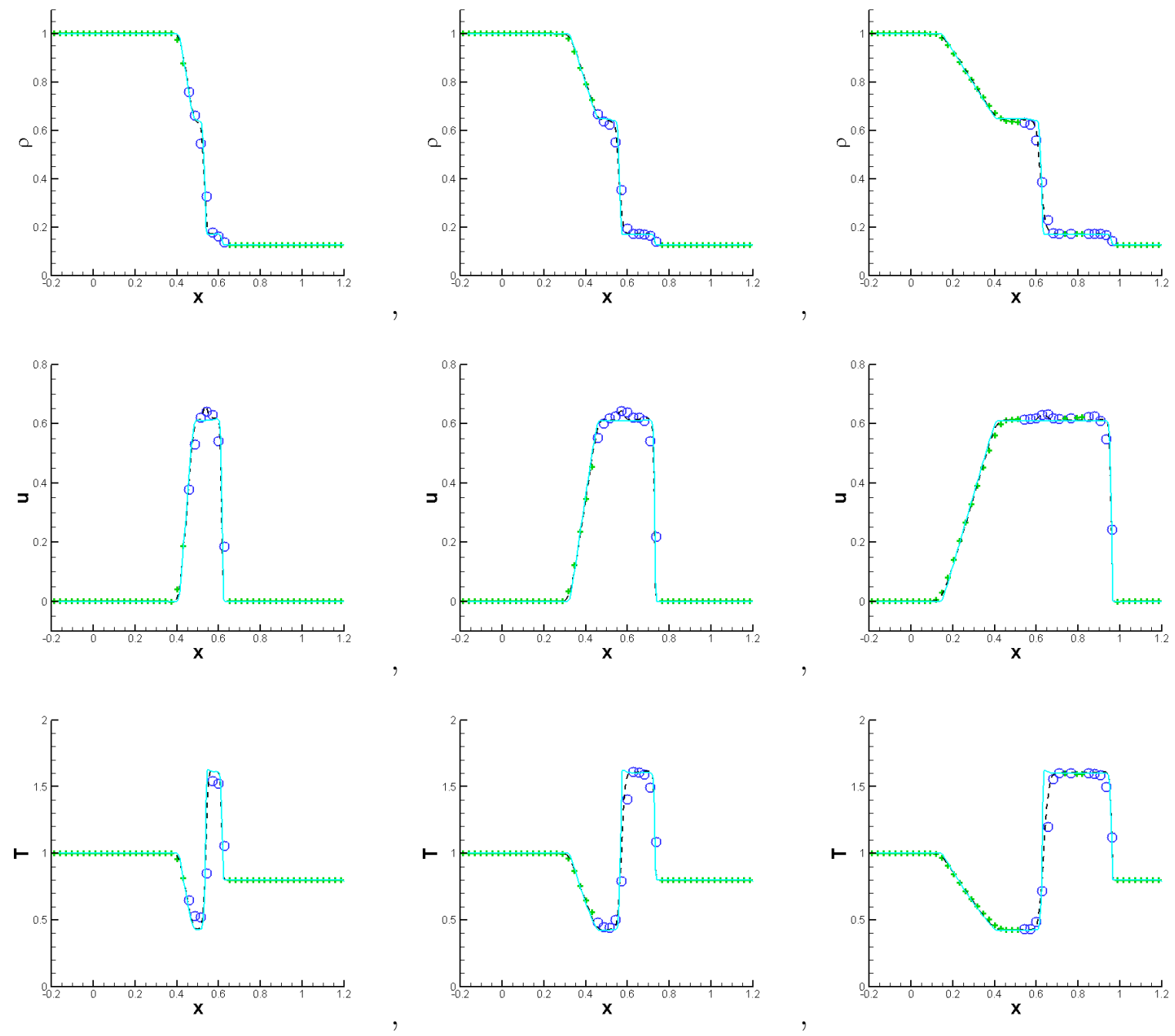

Figure 4.5: Sod problem on the domain $[-0.2,1.2] \times[-4.5,4.5]$. Symbols: $N_{x}=50$ and $N_{v}=100$ with NDG3. From left to right: time $t=0.05,0.1,0.2$. From top to bottom, the density $\rho$, the mean velocity $u$ and the temperature T. Symbols: ' + ' is Euler, circle is kinetic. Solid line: the Euler reference solution. Dashed line: the kinetic reference solution. $\varepsilon=10^{-3}$, Euler-Kinetic.

$\rho$ are computed as follows:

$$
\begin{aligned}
& L^{1} \text { error of } \rho(h)=\frac{1}{2 \pi} \sum_{i} \int_{I_{i}}\left|\rho_{h}(\cdot, T)-\rho_{h / 2}(\cdot, T)\right| d x, \\
& \operatorname{order}=\frac{\log \left(L^{1} \text { error of } \rho(h) / L^{1} \text { error of } \rho(h / 2)\right)}{\log 2},
\end{aligned}
$$

where $\rho_{h}(\cdot, T)$ is the numerical solution of $\rho$ with mesh size $h$ at time $T$. In Table 4.3, we can observe almost 3rd order accuracy. We note that the regime indicators are activated in this test.

Then we take $\varepsilon_{0}=10^{-3}$ and $a_{0}=40$ in $(4.1)$ and $\Omega_{v}=[-10,10]$. In the middle area of $x \in[-0.18,0.18]$, since $\varepsilon=\mathcal{O}(1)$, this part is always identified to be in the kinetic regime. We 

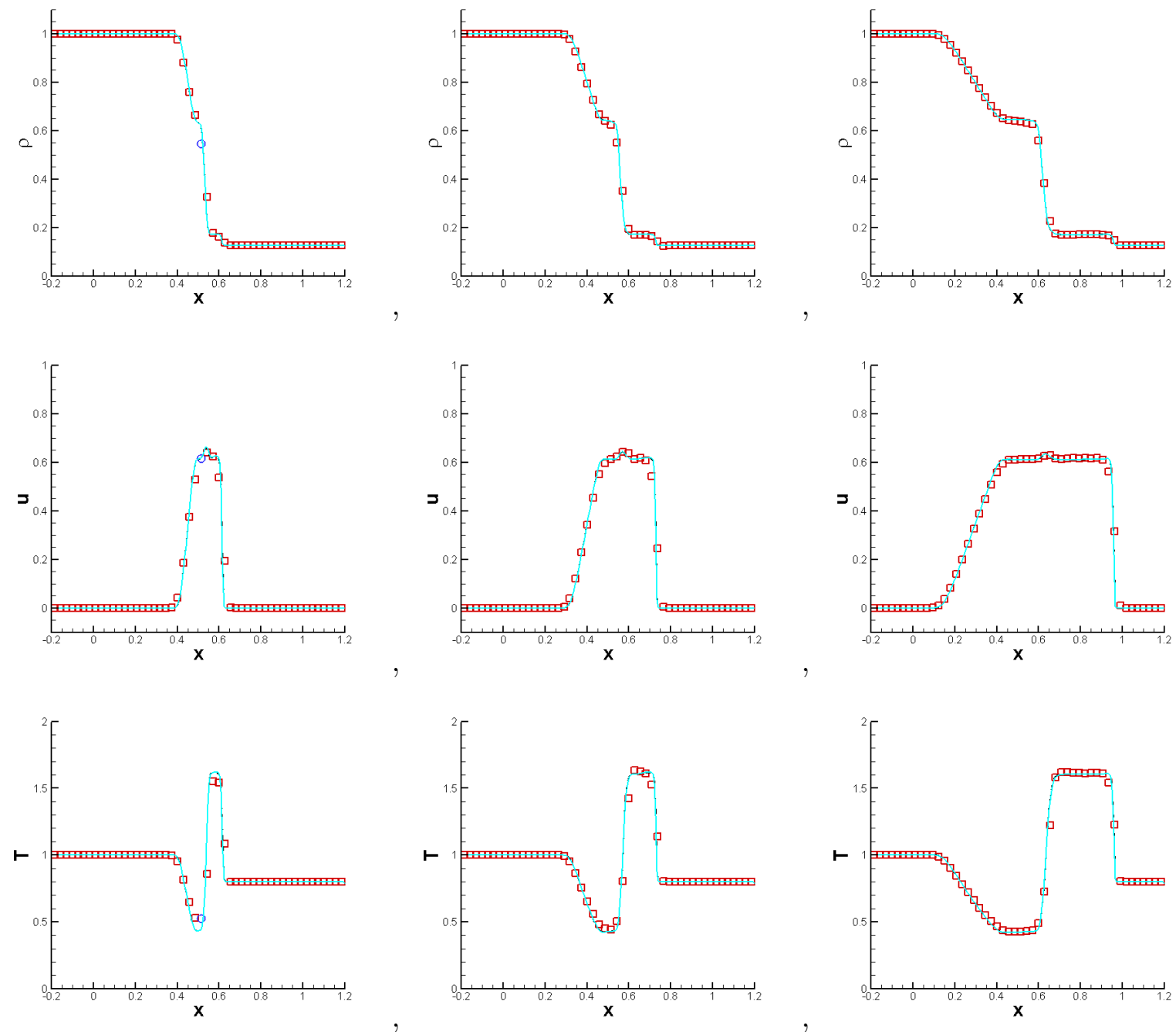

Figure 4.6: Sod problem on the domain $[-0.2,1.2] \times[-4.5,4.5]$. Symbols: $N_{x}=50$ and $N_{v}=100$ with NDG3. From left to right: time $t=0.05,0.1,0.2$. From top to bottom, the density $\rho$, the mean velocity $u$ and the temperature $T$. Symbols: square is NS, circle is kinetic. Solid line: the NS reference solution. Dashed line: the kinetic reference solution. $\varepsilon=10^{-3}$, NS-Kinetic.

report the results in Figures 4.17-4.19 corresponding to Euler-Kinetic, NS-Kinetic and EulerNS-Kinetic schemes, respectively, and in Figure 4.20 for the heat flux. The NS reference solution is obtained under the time step $\Delta t=\mathcal{O}\left(h^{2}\right)$. It is observed that in the middle region, since $\varepsilon(x)=\mathcal{O}(1)$, the NS solution deviates far away from the kinetic reference solution and the heat flux oscillates greatly as can be seen in Figure 4.20. The adaptive algorithm can capture the kinetic reference solution well. For the computational cost, from Table 4.4, we can observe similar result as the blast wave problem for $\varepsilon=10^{-3}$, that is we save $40 \% \sim 45 \%$ of the computational cost for the Euler-NS-Kinetic and NS-Kinetic schemes, but only $20 \%$ for the Euler-Kinetic one. 

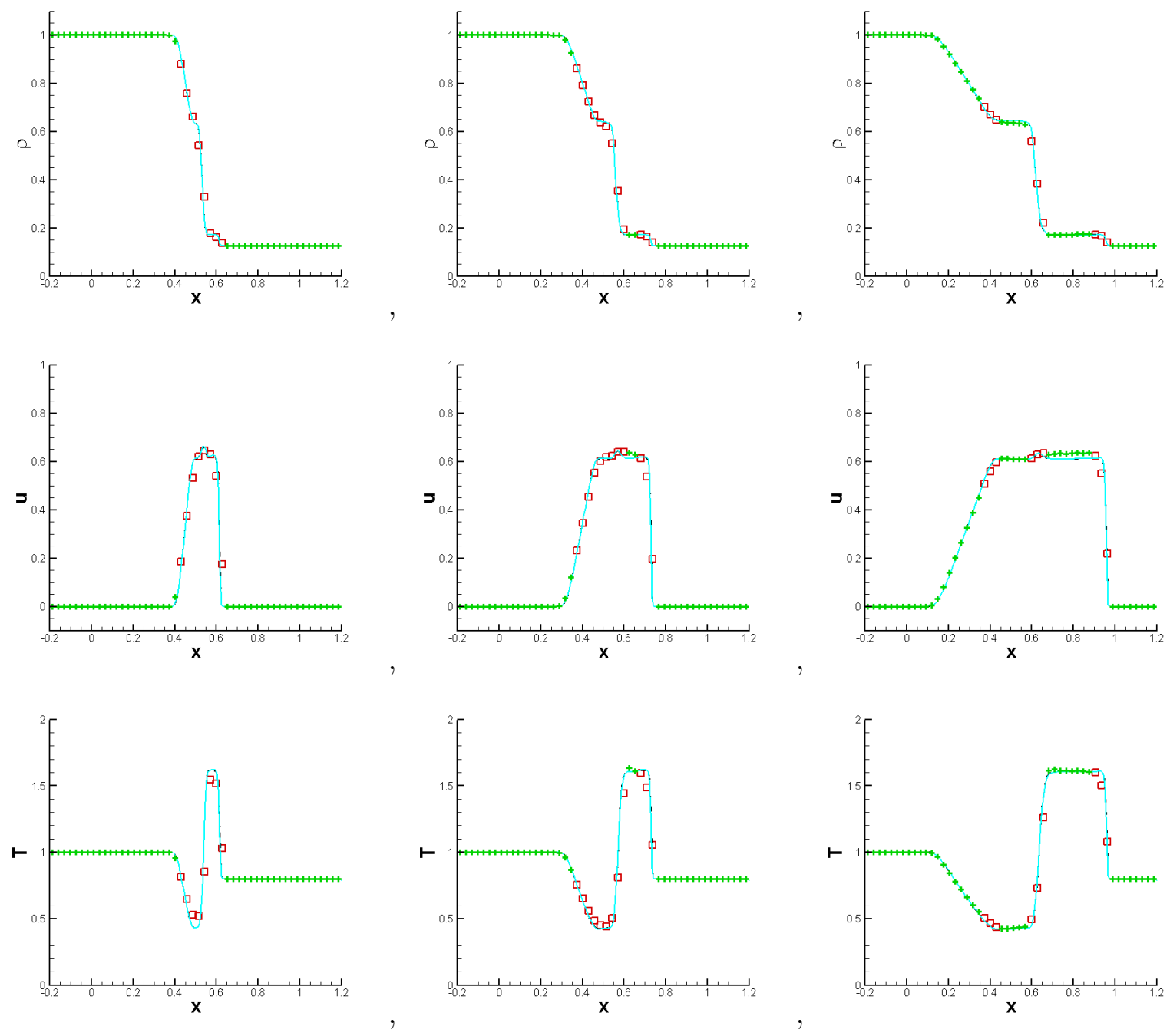

Figure 4.7: Sod problem on the domain $[-0.2,1.2] \times[-4.5,4.5]$. Symbols: $N_{x}=50$ and $N_{v}=100$ with NDG3. From left to right: time $t=0.05,0.1,0.2$. From top to bottom, the density $\rho$, the mean velocity $u$ and the temperature $T$. Symbols: ' + ' is Euler, square is NS, circle is kinetic. Solid line: the NS reference solution. Dashed line: the kinetic reference solution. $\varepsilon=10^{-3}$, Euler-NS-Kinetic.

Table 4.3: $L^{1}$ errors and orders for $\rho$ with $\varepsilon(x)$ in (4.1). $a_{0}=11$ and $\varepsilon_{0}=10^{-6} . T=0.002$. $V_{c}=16$.

\begin{tabular}{|c|c|c|c|c|}
\hline $\mathrm{N}$ & 32 & 64 & 128 & 256 \\
\hline error & $1.69 \mathrm{E}-03$ & $2.44 \mathrm{E}-04$ & $3.41 \mathrm{E}-05$ & $4.79 \mathrm{E}-06$ \\
\hline order & - & 2.79 & 2.84 & 2.83 \\
\hline
\end{tabular}

\section{Conclusion}

We propose a high order hierarchical DG solver for the multi-scale BGK equation. Such hierarchical solver is based on three main ingredients: (1) an asymptotic preserving DG IMEX 

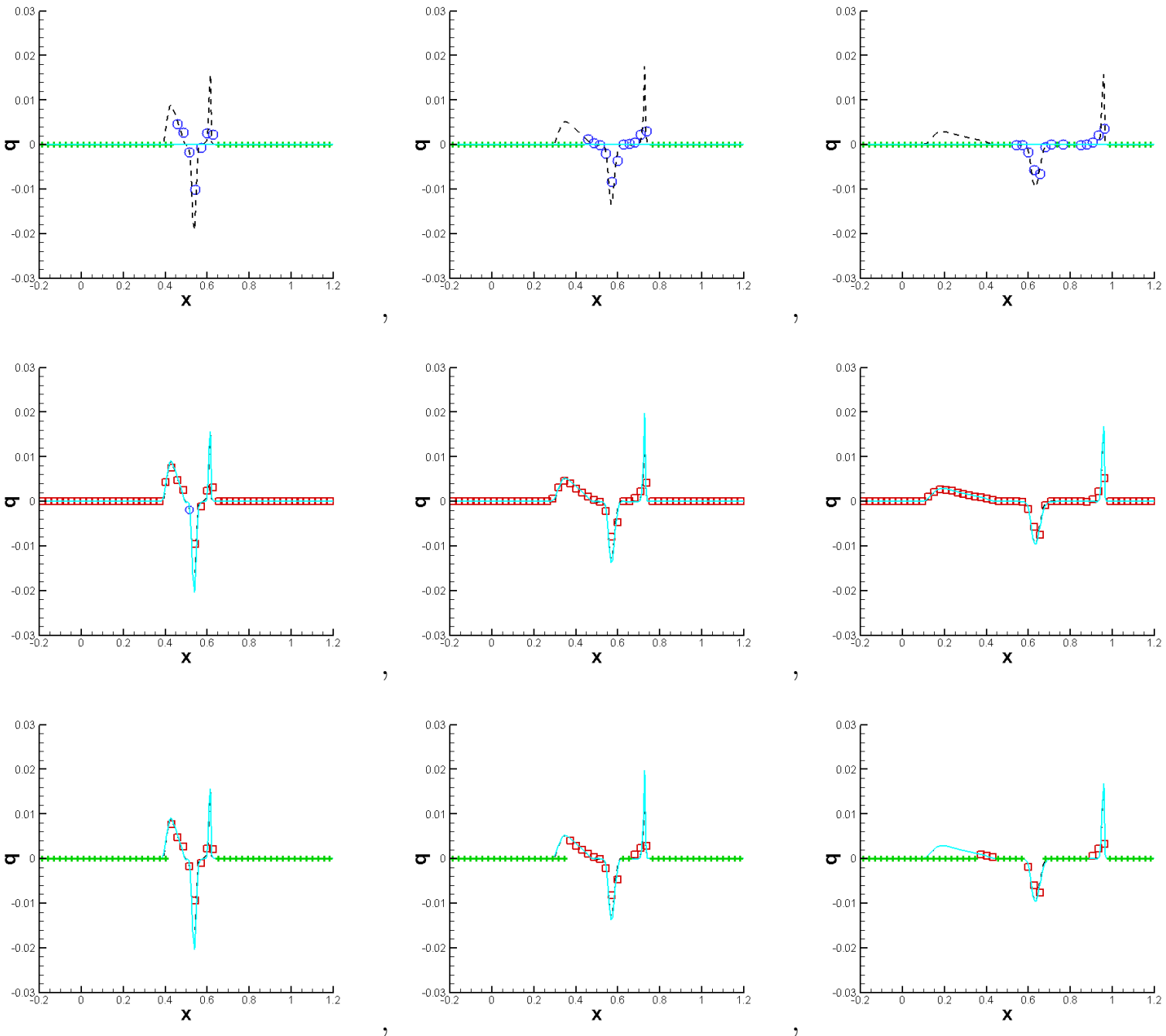

Figure 4.8: Heat flux of sod problem on the domain $[-0.2,1.2] \times[-4.5,4.5]$. Symbols: $N_{x}=50$ and $N_{v}=100$ with NDG3. From left to right: time $t=0.05,0.1,0.2$. From top to bottom, Euler-Kinetic, NS-Kinetic and Euler-NS-Kinetic. Symbols: '+' is Euler, square is NS, circle is kinetic. Solid line: the first row is the Euler reference solution, other two rows are the NS reference solution. Dashed line: the kinetic reference solution. $\varepsilon=10^{-3}$.

Table 4.4: Comparison of the computational cost (seconds) for different indicators. Mixed regime problem.

\begin{tabular}{|c|c|c|c|c|}
\hline method & Euler-NS-Kinetic & NS-Kinetic & Euler-Kinetic & Full kinetic \\
\hline$\varepsilon_{0}=10^{-3}$ & 362.52 & 343.44 & 512.68 & 631.07 \\
\hline
\end{tabular}

scheme originally proposed in [40], which is formally showed to become a DG scheme for the Euler system and a local DG scheme for the Navier-Stokes system; (2) adaptive criteria that automatically switch DG solvers among different regimes for optimal computational effficiency [27]; (3) a compact and seamless coupling conditions between different regimes. Extensive nu- 

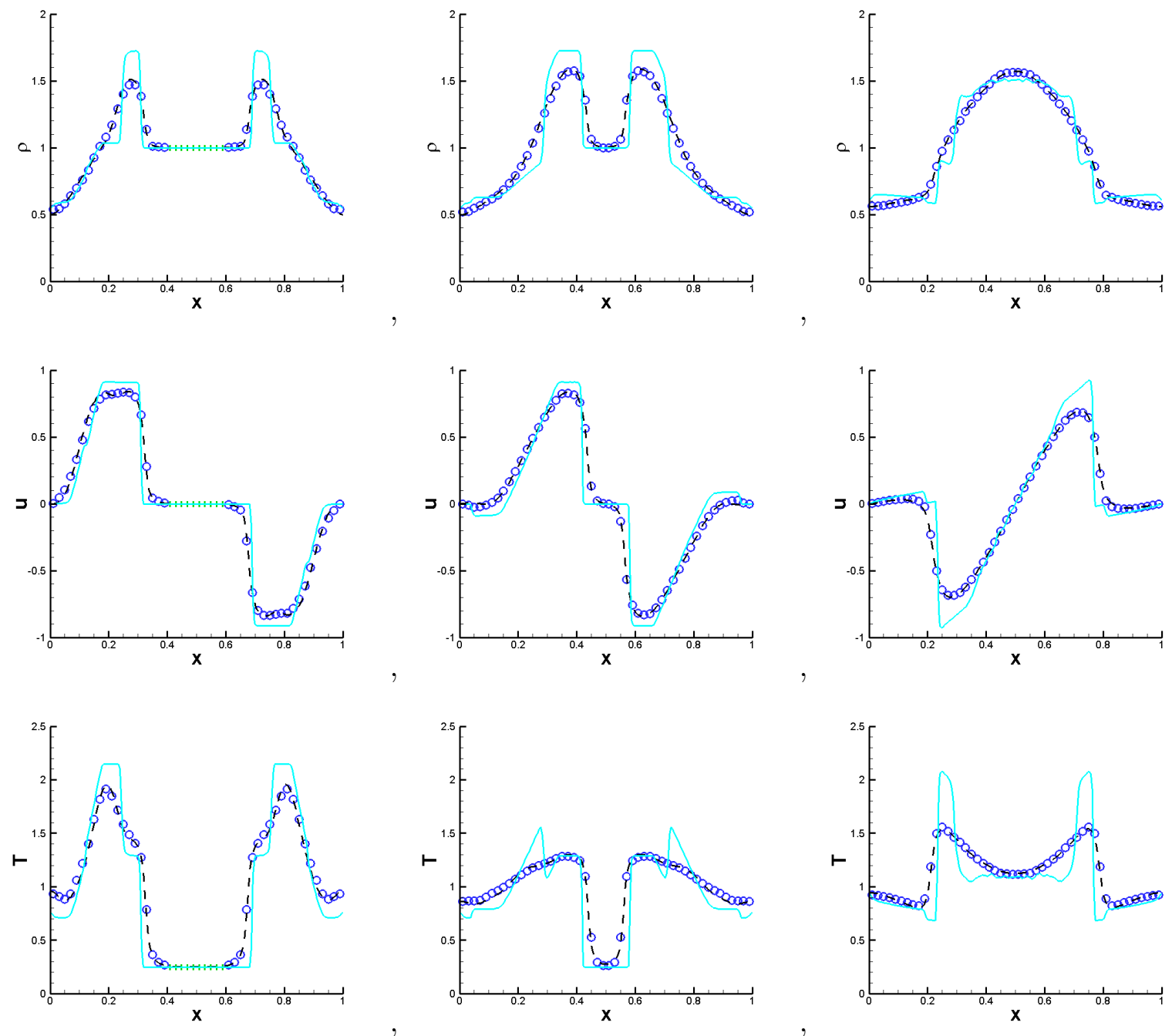

Figure 4.9: Blast wave problem on the domain $[0,1] \times[-9,9]$. Symbols: $N_{x}=50$ and $N_{v}=100$ with NDG3. From left to right: time $t=0.05,0.1,0.25$. From top to bottom, the density $\rho$, the mean velocity $u$ and the temperature $T$. Symbols: ' + ' is Euler, circle is kinetic. Solid line: the Euler reference solution. Dashed line: the kinetic reference solution. $\varepsilon=10^{-2}$, Euler-Kinetic.

merical experiments are performed to showcase the proposed scheme in its ability for capturing solution structures and in computational savings.

\section{References}

[1] A. Alaia And G. Puppo, A hybrid method for hydrodynamic-kinetic flow, Part II: Coupling of hydrodynamic and kinetic models, Journal of Computational Physics, 232 (2012), pp. $5217-5242$.

[2] P. Andries, P. L. Tallec, J. P. Perlat, and B. Perthame, The Gaussian-BGK model of Boltzmann equation with small Prandtl number, European Journal of Mechanics - B/Fluids, 19 (2000), pp. 813 - 830. 

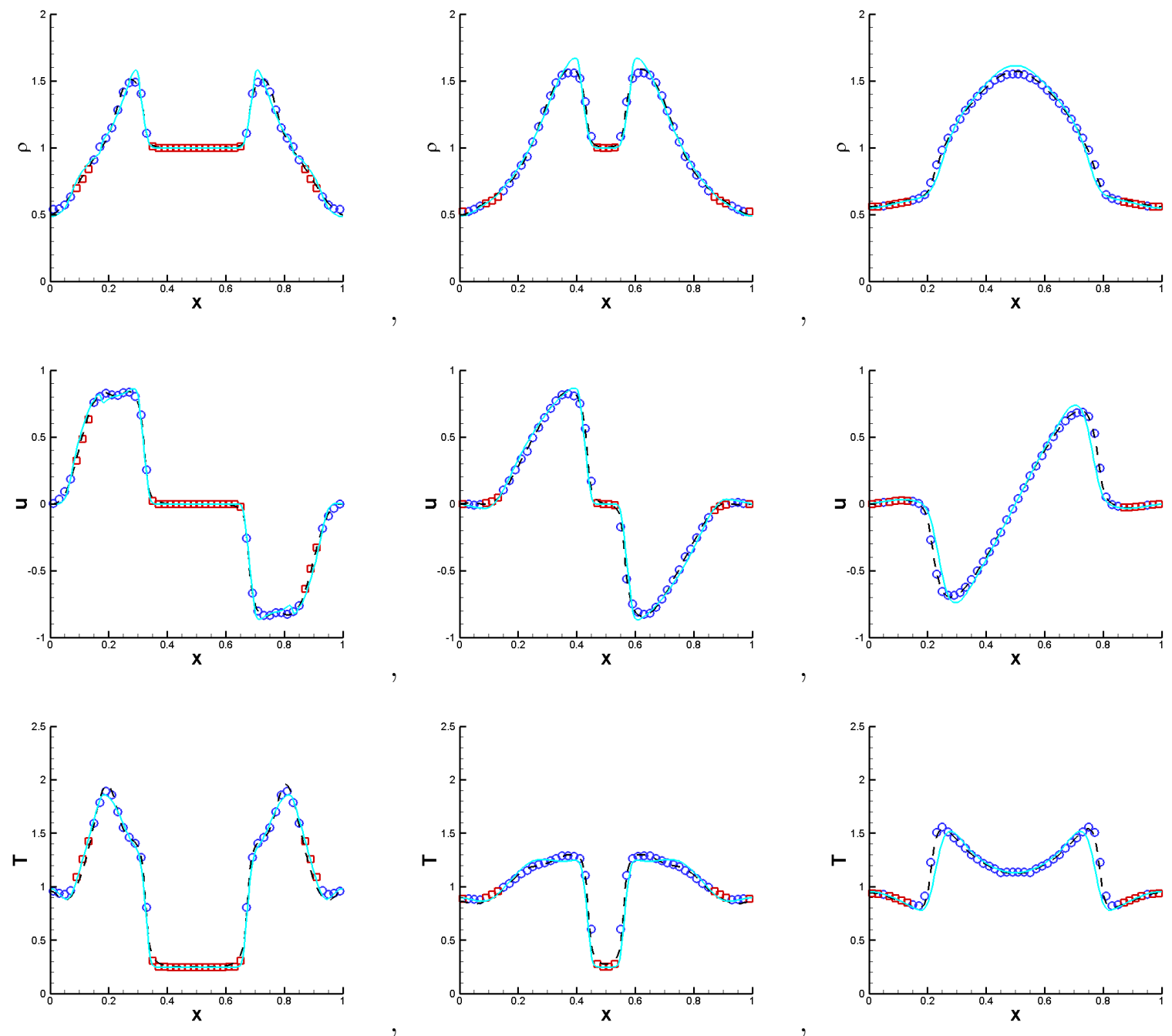

Figure 4.10: Blast wave problem on the domain $[0,1] \times[-9,9]$. Symbols: $N_{x}=50$ and $N_{v}=100$ with NDG3. From left to right: time $t=0.05,0.1,0.25$. From top to bottom, the density $\rho$, the mean velocity $u$ and the temperature $T$. Symbols: square is NS, circle is kinetic. Solid line: the NS reference solution. Dashed line: the kinetic reference solution. $\varepsilon=10^{-2}$, NS-Kinetic.

[3] U. Ascher, S. Ruuth, And R. Spiteri, Implicit-explicit Runge-Kutta methods for time-dependent partial differential equations, Applied Numerical Mathematics, 25 (1997), pp. 151-167.

[4] C. Bardos, F. Golse, and D. Levermore, Fluid dynamic limits of kinetic equations. I. Formal derivations, Journal of Statistical Physics, 63 (1991), pp. 323-344.

[5] F. Bassi And S. ReBay, A high-order accurate discontinuous finite element method for the numerical solution of the compressible Navier-Stokes equations, Journal of Computational Physics, 131 (1997), pp. 267-279.

[6] F. Bassi And S. Rebay, Numerical evaluation of two discontinuous Galerkin methods for the compressible Navier-Stokes equations, International Journal for Numerical Methods in 

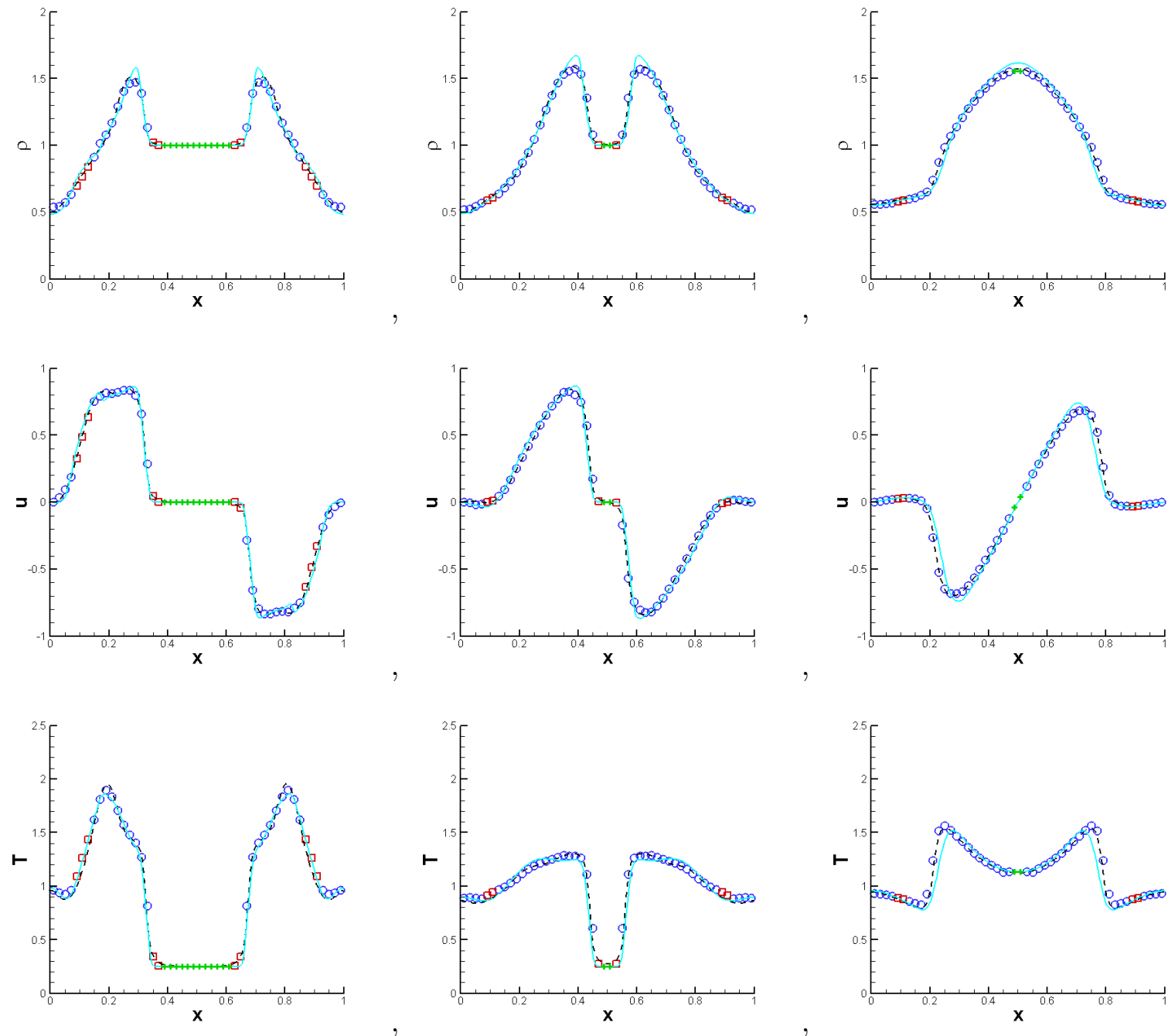

Figure 4.11: Blast wave problem on the domain $[0,1] \times[-9,9]$. Symbols: $N_{x}=50$ and $N_{v}=100$ with NDG3. From left to right: time $t=0.05,0.1,0.25$. From top to bottom, the density $\rho$, the mean velocity $u$ and the temperature T. Symbols: ' + ' is Euler, square is NS, circle is kinetic. Solid line: the NS reference solution. Dashed line: the kinetic reference solution. $\varepsilon=10^{-2}$, Euler-NS-Kinetic.

Fluids, 40 (2002), pp. 197-207.

[7] C. E. Baumann and J. T. Oden, A discontinuous hp finite element method for the Euler and Navier-Stokes equations, International Journal for Numerical Methods in Fluids, 31 (1999), pp. 79-95.

[8] M. Bennoune, M. Lemou, And L. Mieussens, Uniformly stable numerical schemes for the Boltzmann equation preserving the compressible Navier-Stokes asymptotics, Journal of Computational Physics, 227 (2008), pp. 3781-3803.

[9] P. L. Bhatnagar, E. P. Gross, and M. Krook, A model for collision processes in gases. I. Small amplitude processes in charged and neutral one-component systems, Physical 

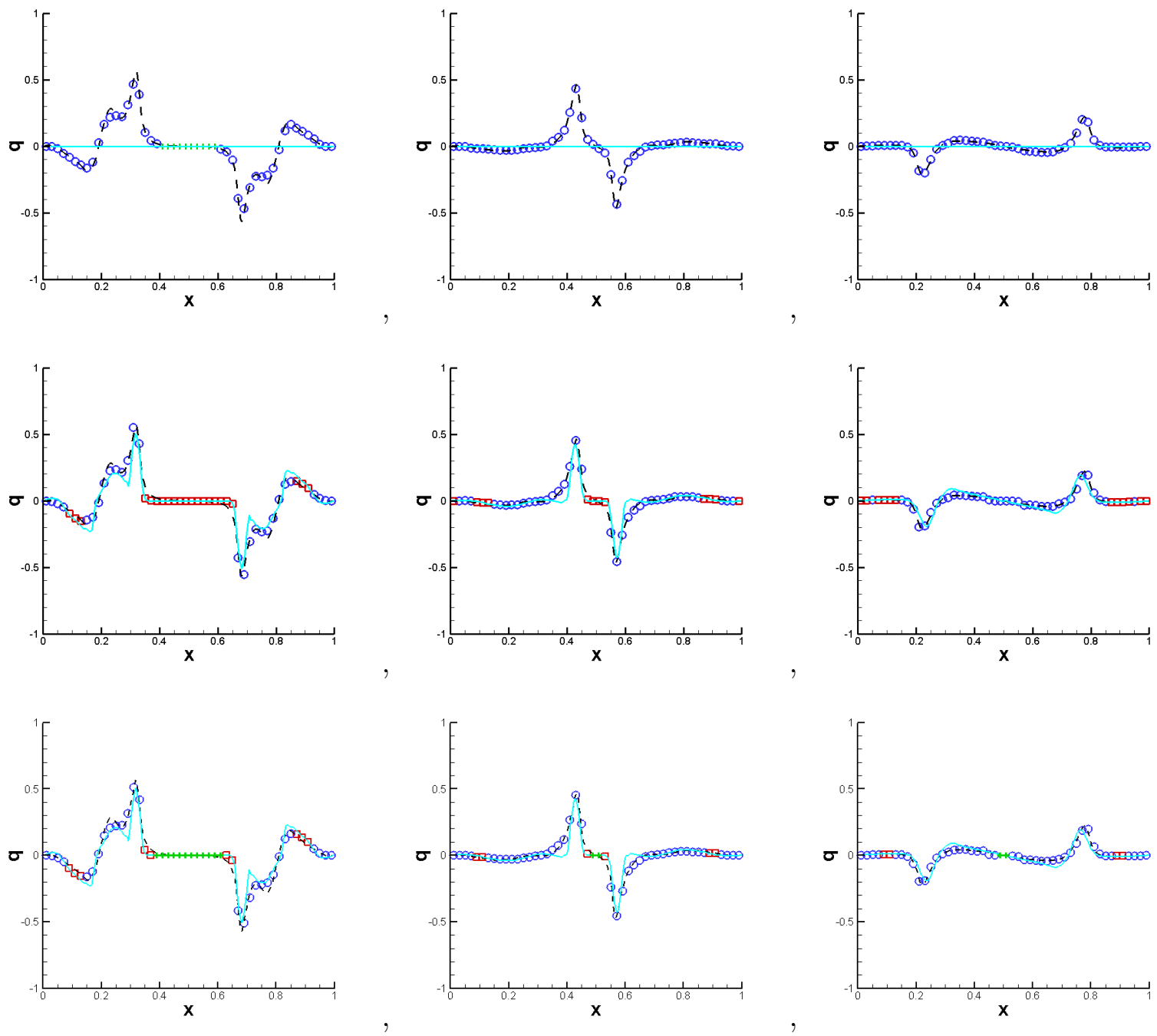

Figure 4.12: Heat flux of the blast wave problem on the domain $[0,1] \times[-9,9]$. Symbols: $N_{x}=50$ and $N_{v}=100$ with NDG3. From left to right: time $t=0.05,0.1,0.2$. From top to bottom, Euler-Kinetic, NS-Kinetic and Euler-NS-Kinetic. Symbols: ' + ' is Euler, square is NS, circle is kinetic. Solid line: the first row is the Euler reference solution, other two rows are the NS reference solution. Dashed line: the kinetic reference solution. $\varepsilon=10^{-2}$.

Review, 94 (1954), p. 511.

[10] S. Boscarino, L. Pareschi, and G. Russo, Implicit-Explicit Runge-Kutta Schemes for Hyperbolic Systems and Kinetic Equations in the Diffusion Limit, SIAM Journal on Scientific Computing, 35 (2013), pp. A22-A51.

[11] S. Boscarino And G. Russo, On a class of uniformly accurate IMEX Runge-Kutta schemes and applications to hyperbolic systems with relaxation, SIAM Journal on Scientific Computing, 31 (2010), pp. 1926-1945.

[12] I. D. Boyd, G. Chen, And G. V. Candler, Predicting failure of the continuum fluid equations in transitional hyperbolic flows, Physical Fluids, 7 (1995), pp. 210 - 219. 

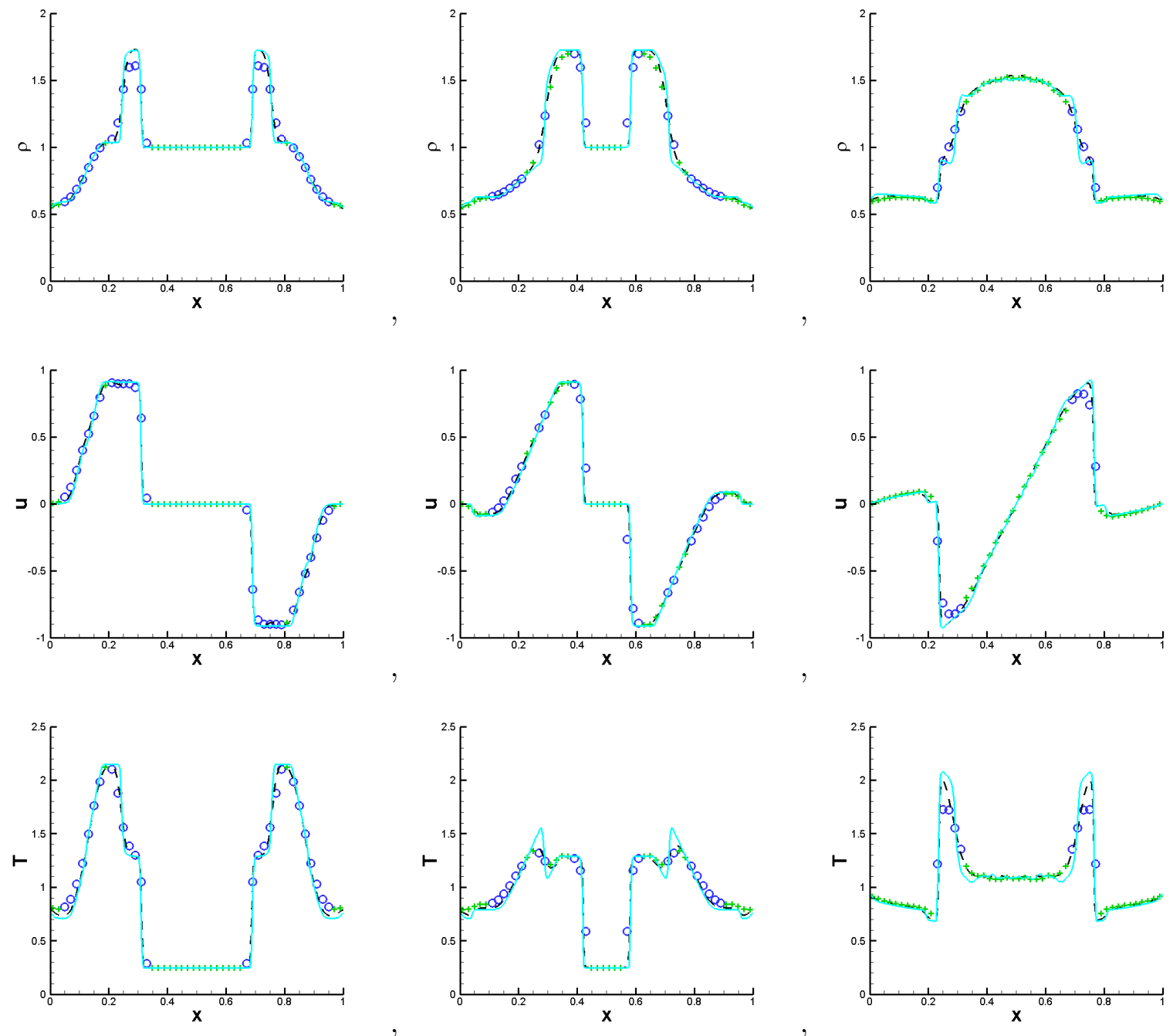

Figure 4.13: Blast wave problem on the domain $[0,1] \times[-9,9]$. Symbols: $N_{x}=50$ and $N_{v}=100$ with NDG3. From left to right: time $t=0.05,0.1,0.25$. From top to bottom, the density $\rho$, the mean velocity $u$ and the temperature T. Symbols: ' + ' is Euler, square is NS, circle is kinetic. Solid line: the NS reference solution. Dashed line: the kinetic reference solution. $\varepsilon=10^{-3}$, Euler-Kinetic.

[13] J. P. Boyd, Chebyshev and Fourier spectral methods, Courier Dover Publications, 2001.

[14] C. Cercignani, Mathematical methods in kinetic theory, Springer, 1969.

[15] _ _ The Boltzmann equation, Springer, 1988.

[16] __ Rarefied gas dynamics: from basic concepts to actual calculations, vol. 21, Cambridge University Press, 2000.

[17] B. CockBurn, S.-Y. Lin, And C.-W. Shu, TVB Runge-Kutta local projection discontinuous Galerkin finite element method for conservation laws III: one-dimensional systems, Journal of Computational Physics, 84 (1989), pp. 90-113. 

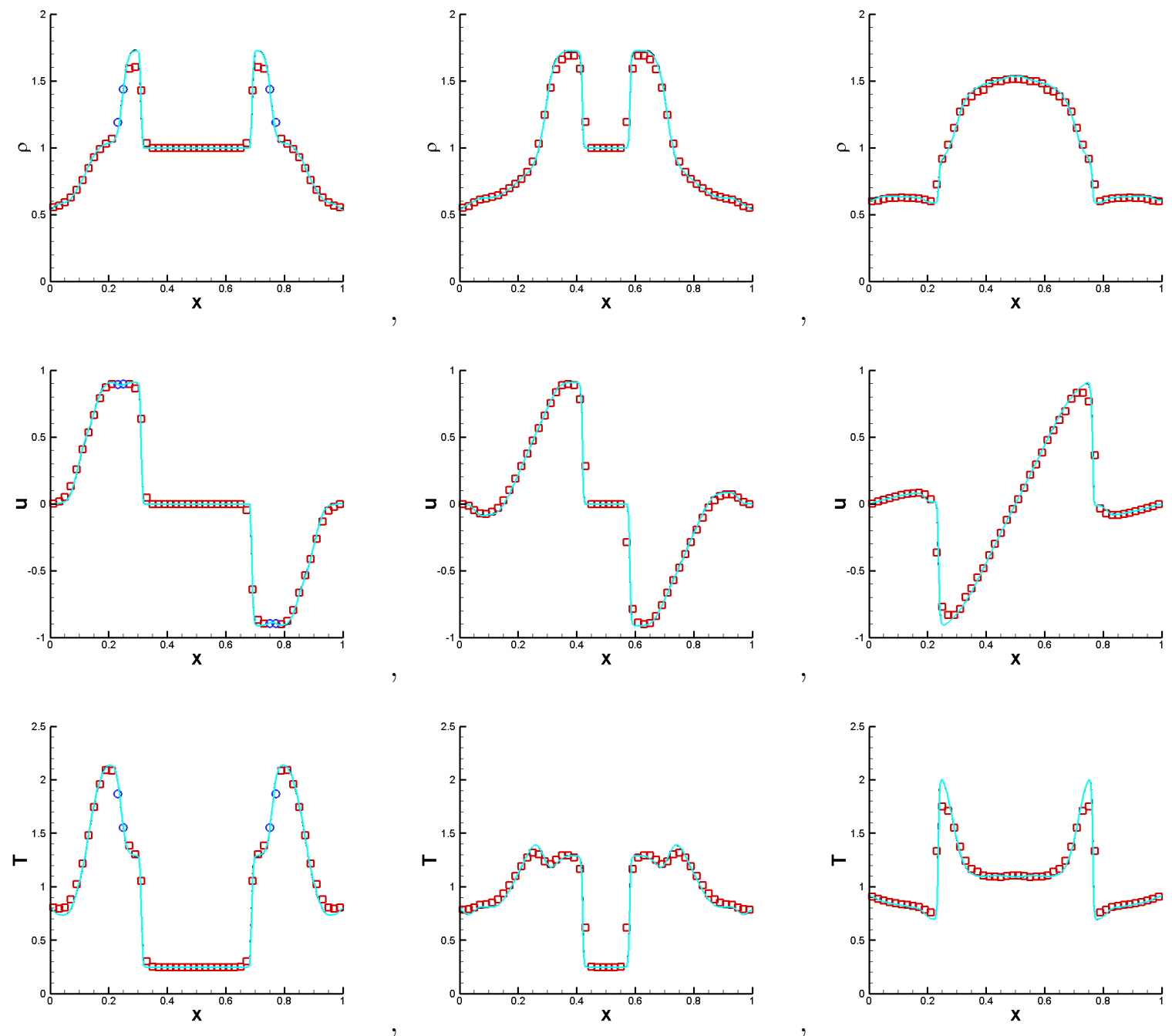

Figure 4.14: Blast wave problem on the domain $[0,1] \times[-9,9]$. Symbols: $N_{x}=50$ and $N_{v}=100$ with NDG3. From left to right: time $t=0.05,0.1,0.25$. From top to bottom, the density $\rho$, the mean velocity $u$ and the temperature T. Symbols: ' + ' is Euler, square is NS, circle is kinetic. Solid line: the NS reference solution. Dashed line: the kinetic reference solution. $\varepsilon=10^{-3}$, NS-Kinetic.

[18] B. Cockburn And C.-W. Shu, The local discontinuous Galerkin method for timedependent convection-diffusion systems, SIAM Journal on Numerical Analysis, 35 (1998), pp. 2440-2463.

[19] P. Degond and G. Dimarco, Fluid simulations with localized Boltzmann upscaling by direct simulation Monte-Carlo, Journal of Computational Physics, 231 (2012), pp. 2414 2437.

[20] P. Degond, G. Dimarco, and L. Mieussens, A moving interface method for dynamic kinetic-fluid coupling, Journal of Computational Physics, 227 (2007), pp. 1176-1208. 

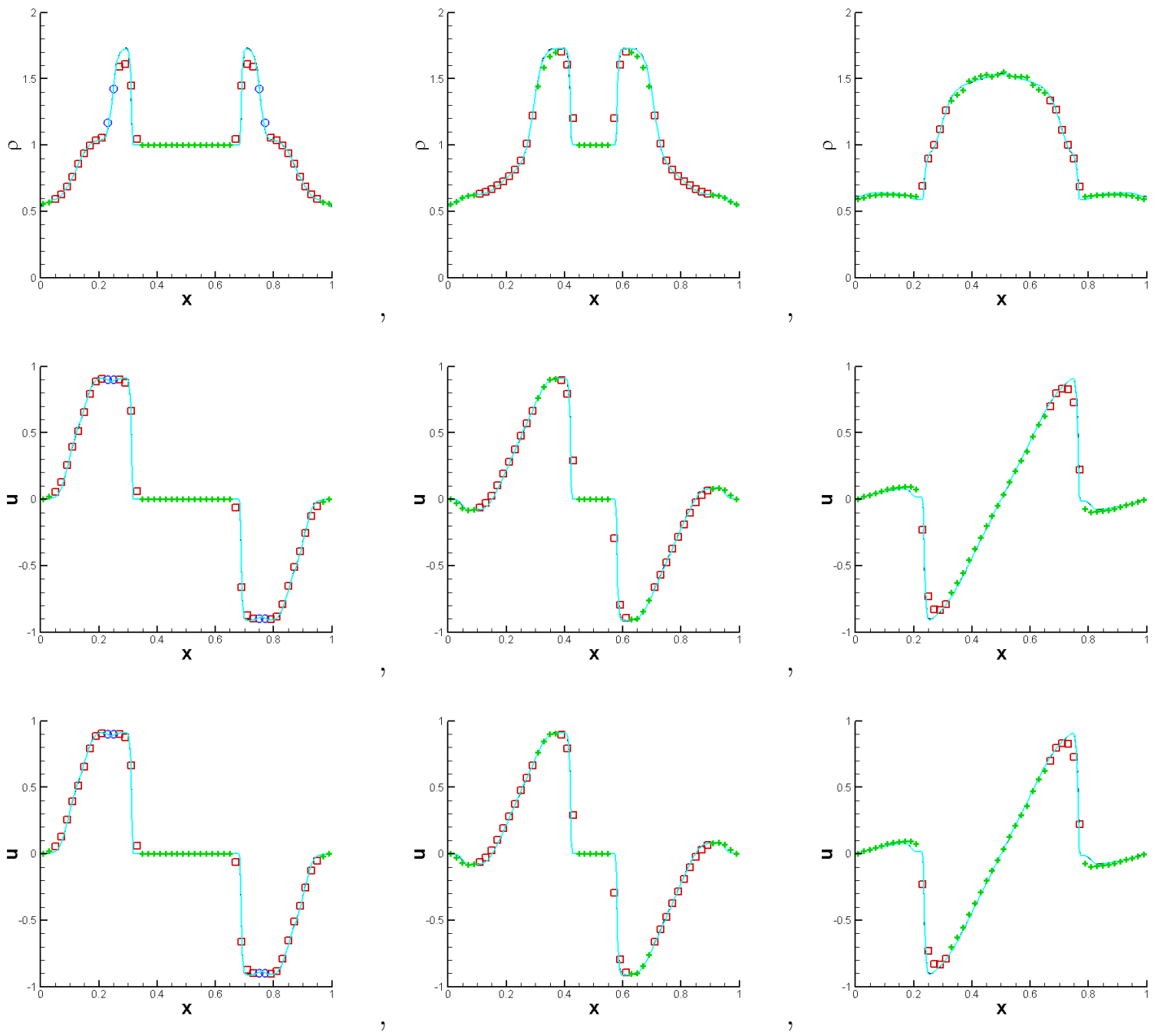

Figure 4.15: Blast wave problem on the domain $[0,1] \times[-9,9]$. Symbols: $N_{x}=50$ and $N_{v}=100$ with NDG3. From left to right: time $t=0.05,0.1,0.25$. From top to bottom, the density $\rho$, the mean velocity $u$ and the temperature T. Symbols: ' + ' is Euler, square is NS, circle is kinetic. Solid line: the NS reference solution. Dashed line: the kinetic reference solution. $\varepsilon=10^{-3}$, Euler-NS-Kinetic.

[21] P. Degond, G. Dimarco, and L. Mieussens, A multiscale kinetic-fluid solver with $d y$ namic localization of kinetic effects, Journal of Computational Physics, 229 (2010), pp. 4907 $-4933$.

[22] P. Degond, S. Jin, And L. Mieussens, A smooth transition model between kinetic and hydrodynamic equations, Journal of Computational Physics, 209 (2005), pp. 665-694.

[23] P. Degond, J.-G. Liu, And L. Mieussens, Macroscopic fluid models with localized kinetic upscaling effects, Multiscale Modeling \& Simulation, 5 (2006), pp. 940-979.

[24] G. Dimarco and L. Pareschi, Fluid solver independent hybrid methods for multiscale kinetic equations, SIAM Journal on Scientific Computing, 32 (2010), pp. 603-634. 

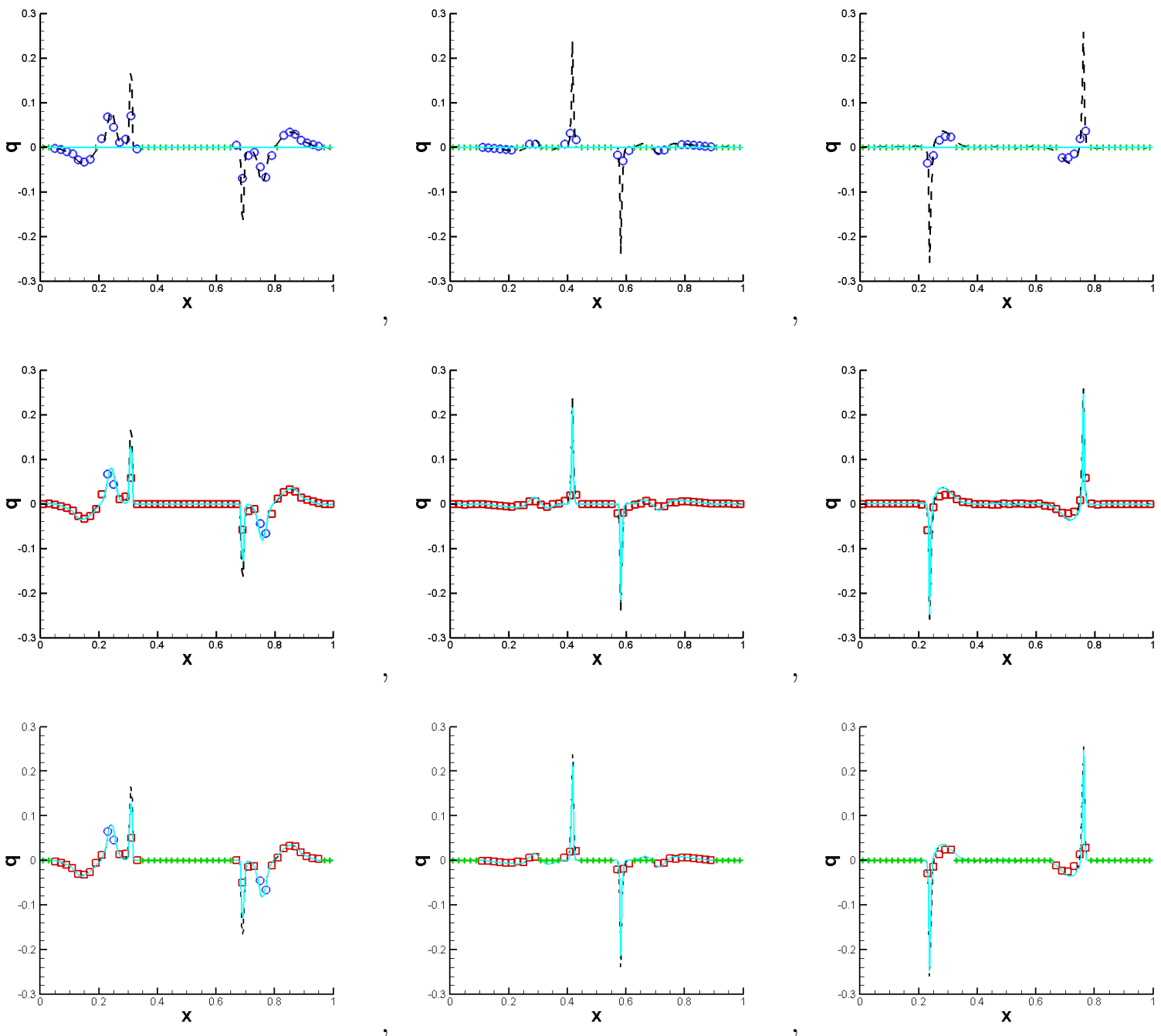

Figure 4.16: Heat flux of the blast wave problem on the domain $[0,1] \times[-9,9]$. Symbols: $N_{x}=50$ and $N_{v}=100$ with NDG3. From left to right: time $t=0.05,0.1,0.2$. From top to bottom, Euler-Kinetic, NS-Kinetic and Euler-NS-Kinetic. Symbols: ' + ' is Euler, square is NS, circle is kinetic. Solid line: the first row is the Euler reference solution, other two rows are the NS reference solution. Dashed line: the kinetic reference solution. $\varepsilon=10^{-3}$.

[25] F. Filbet And S. Jin, A class of asymptotic-preserving schemes for kinetic equations and related problems with stiff sources, Journal of Computational Physics, 229 (2010), pp. $7625-7648$.

[26] — - An asymptotic preserving scheme for the ES-BGK model of the Boltzmann equation, Journal of Scientific Computing, 46 (2011), pp. 204-224.

[27] F. Filbet And T. Rey, A hierarchy of hybrid numerical methods for multiscale kinetic equations, SIAM Journal on Scientific Computing, 37 (2015), pp. A1218 - A1247.

[28] J. S. Hesthaven and T. Warburton, Nodal discontinuous Galerkin methods: algorithms, analysis, and applications, vol. 54, Springerverlag New York, 2008. 

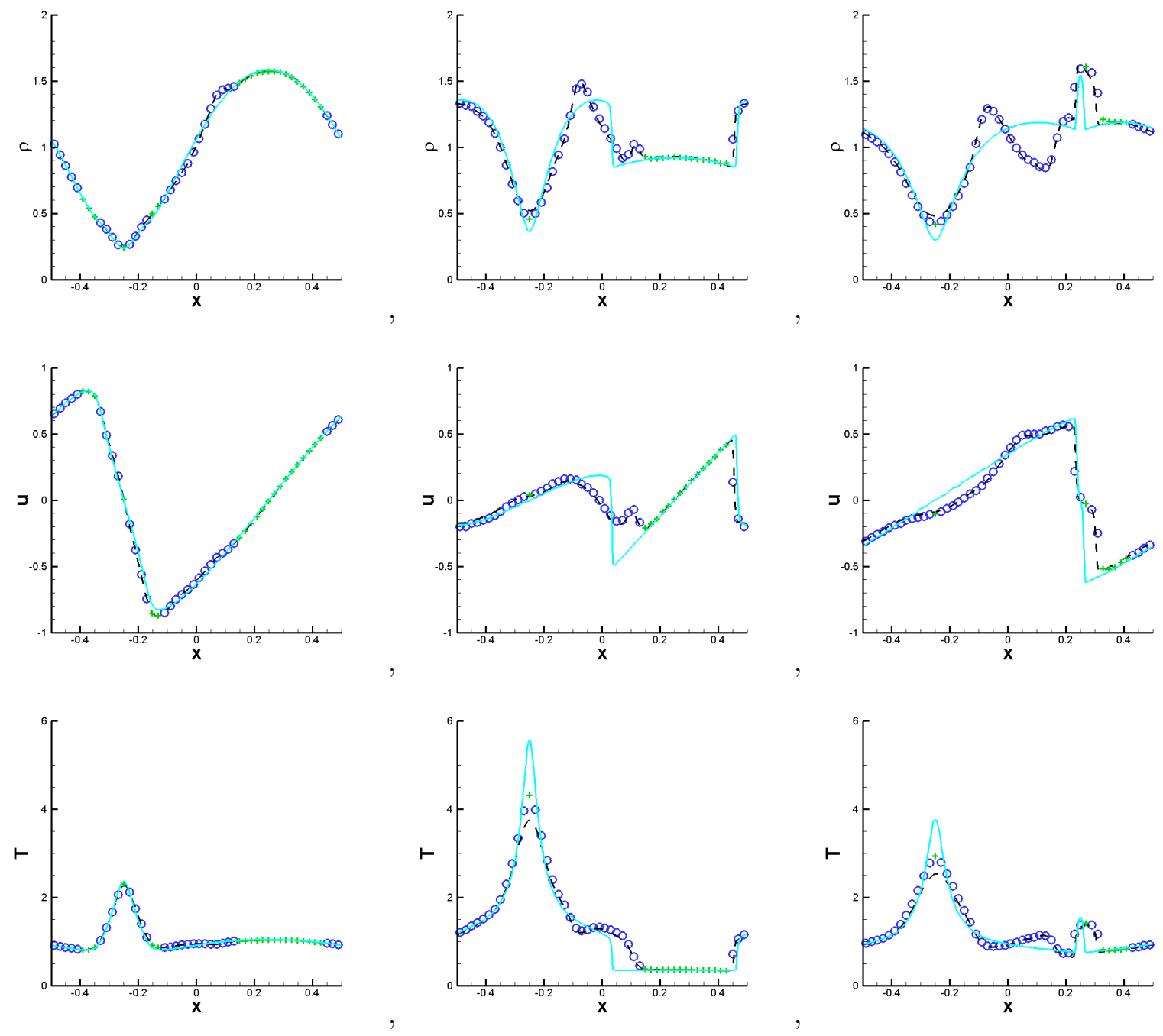

Figure 4.17: Mixed regime problem with $\varepsilon(x)$ with $a_{0}=40$ on the domain $[-0.5,0.5] \times[-10,10]$. $N_{x}=50$ and $N_{v}=100$ with NDG3. Symbols: ' + ' is Euler, circle is kinetic. Solid line: the reference Euler solution with $N_{x}=100$ and $N_{v}=100$. Dashed line: the reference kinetic solution with $N_{x}=100$ and $N_{v}=100$. From left to right: time $t=0.1,0.3,0.45$. From top to bottom, the density $\rho$, the mean velocity $u$ and the temperature T. $\varepsilon_{0}=10^{-3}$, Euler-Kinetic.

[29] S. JIn, Asymptotic preserving (AP) schemes for multiscale kinetic and hyperbolic equations: a review, Lecture Notes for Summer School on Methods and Models of Kinetic Theory (M\&MKT), Porto Ercole (Grosseto, Italy), (2010).

[30] C. D. Levermore, W. J. Morokoff, and B. T. Nadiga, Moment realizability and the validity of the Navier-Stokes equations for rarefied gas dynamics, Physics of Fluids, 10 (1998), pp. $3214-3226$.

[31] I. Lomtev and G. E. Karniadakis, A discontinuous Galerkin method for the NavierStokes equations, International Journal for Numerical Methods in Fluids, 29 (1999), pp. 587-603. 

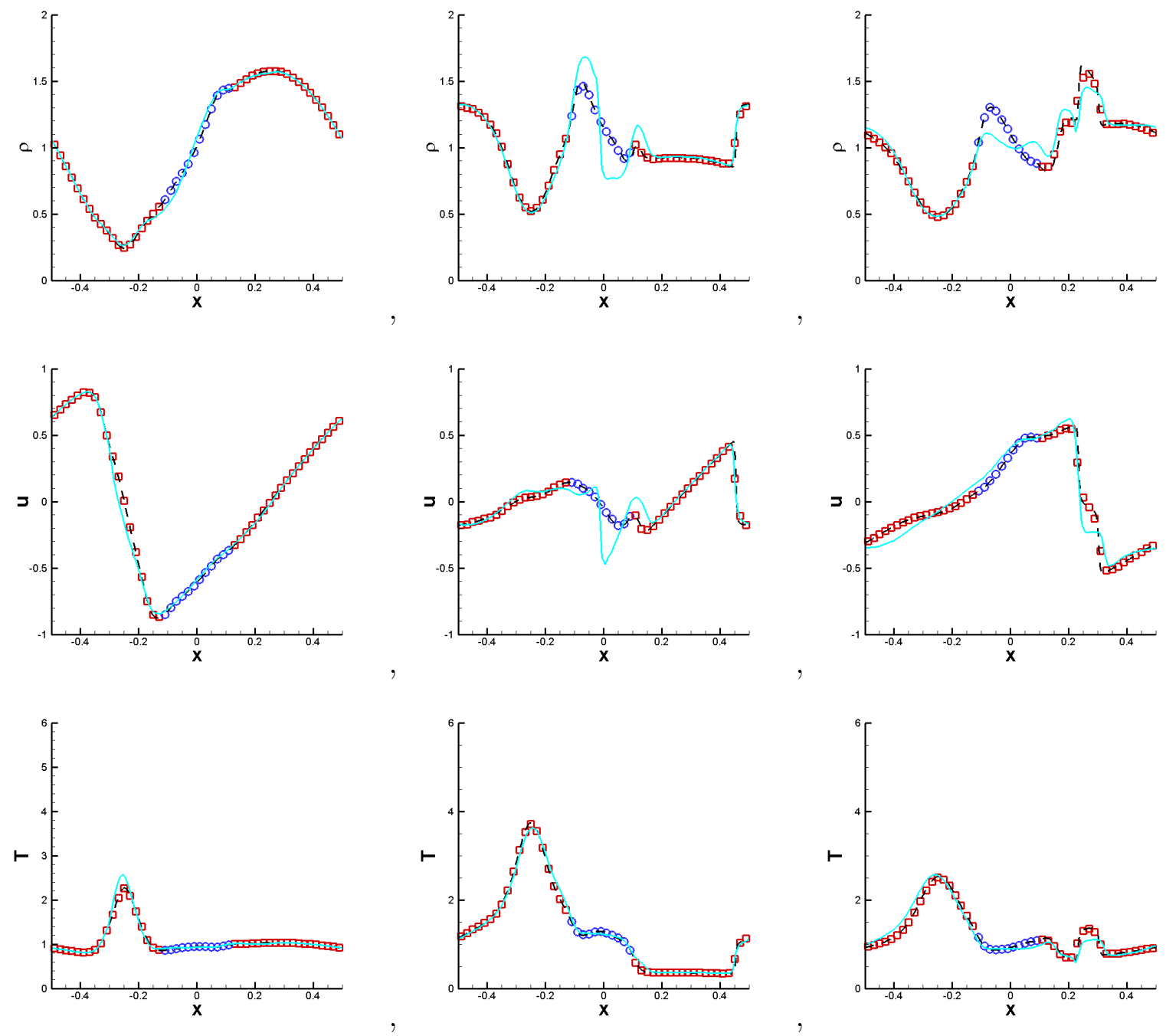

Figure 4.18: Mixed regime problem with $\varepsilon(x)$ with $a_{0}=40$ on the domain $[-0.5,0.5] \times[-10,10]$. $N_{x}=50$ and $N_{v}=100$ with NDG3. Symbols: square is NS, circle is kinetic. Solid line: the reference NS solution with $N_{x}=100$ and $N_{v}=100$. Dashed line: the reference kinetic solution with $N_{x}=100$ and $N_{v}=100$. From left to right: time $t=0.1,0.3,0.45$. From top to bottom, the density $\rho$, the mean velocity $u$ and the temperature T. $\varepsilon_{0}=10^{-3}$, NS-Kinetic.

[32] L. Mieussens, Discrete-velocity models and numerical schemes for the Boltzmann-BGK equation in plane and axisymmetric geometries, Journal of Computational Physics, 162 (2000), pp. 429-466.

[33] S. Pieraccini and G. Puppo, Implicit-explicit schemes for BGK kinetic equations, Journal of Scientific Computing, 32 (2007), pp. 1-28.

[34] — Microscopically implicit-macroscopically explicit schemes for the BGK equation, Journal of Computational Physics, 231 (2012), pp. 299-327.

[35] C.-W. Shu, Tvb uniformly high-order schemes for conservation laws, Mathematics of Computation, 49 (1987), pp. 105-121. 

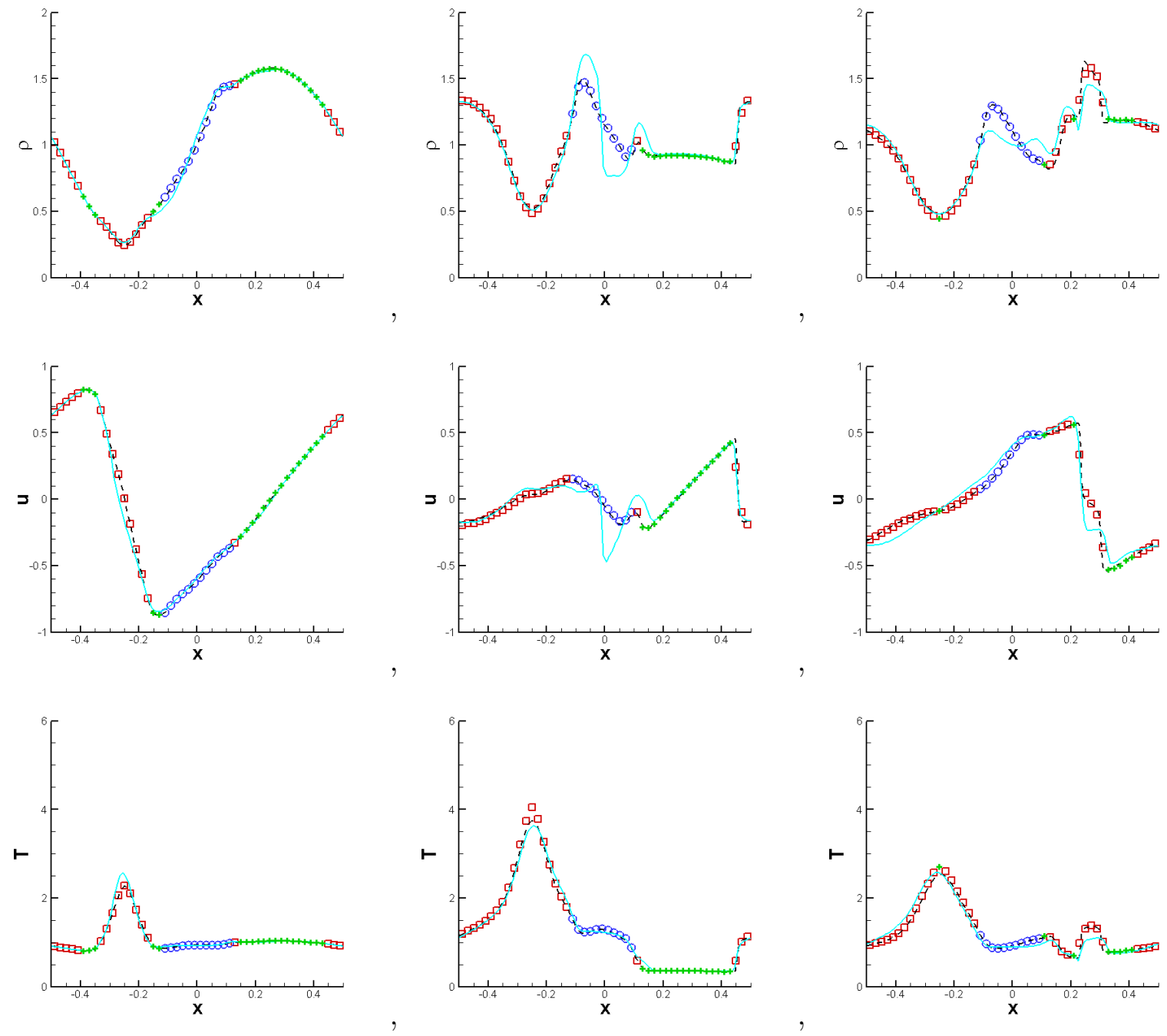

Figure 4.19: Mixed regime problem with $\varepsilon(x)$ with $a_{0}=40$ on the domain $[-0.5,0.5] \times[-10,10]$. $N_{x}=50$ and $N_{v}=100$ with NDG3. Symbols: ' + ' is Euler, square is NS, circle is kinetic. Solid line: the reference NS solution with $N_{x}=100$ and $N_{v}=100$. Dashed line: the reference kinetic solution with $N_{x}=100$ and $N_{v}=100$. From left to right: time $t=0.1,0.3,0.45$. From top to bottom, the density $\rho$, the mean velocity $u$ and the temperature $T$. $\varepsilon_{0}=10^{-3}$, Euler-NS-Kinetic.

[36] _ _ High-order finite difference and finite volume WENO schemes and discontinuous Galerkin methods for CFD, International Journal of Computational Fluid Dynamics, 17 (2003), pp. 107-118.

[37] S. TIWARI, Coupling of the Boltzmann and Euler equations with automatic domain decomposition, Journal of Computational Physics, 144 (1998), pp. 710 - 726.

[38] S. Tiwari, A. Klar, AND S. HARDT, Simulations of micro channel gas flows with domain decomposition technique for kinetic and fluid dynamic equations, in Domain Decomposition Methods in Science and Engineering XXI, Springer, Cham, Switzerland, 2012, pp. $197-206$. 

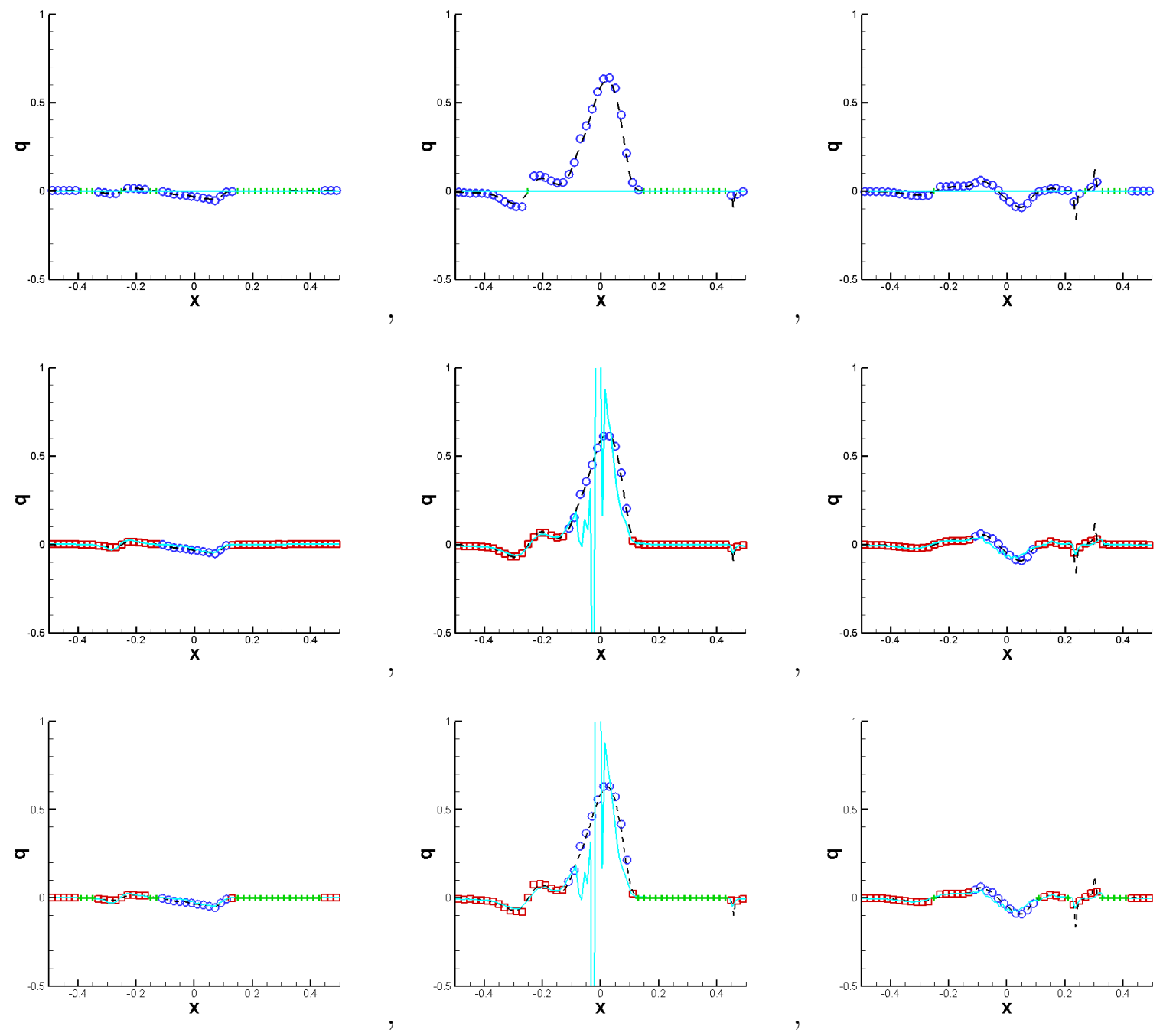

Figure 4.20: Heat flux of the mixed regime problem on the domain $[-0.5,0.5] \times[-10,10]$. Symbols: ' + ' is Euler, square is NS, circle is kinetic. Solid line: the first row is the Euler reference solution, other two rows are the reference NS solution, with $N_{x}=100$ and $N_{v}=100$. Dashed line: the reference kinetic solution with $N_{x}=100$ and $N_{v}=100$. From left to right: time $t=0.1,0.3,0.45 . \varepsilon_{0}=10^{-3}$.

[39] — - A particle-particle hybrid method for kinetic and continuum equations, Journal of Computational Physics, 228 (2009), pp. 7109 - 7124.

[40] T. XIOng, J. JAng, F. LI, AND J.-M. QIU, High order asymptotic preserving nodal discontinuous Galerkin IMEX schemes for the BGK equation, Journal of Computational Physics, 284 (2015), pp. 70-94. 